\title{
On the Ultimate Tensile Strength of Tantalum
}

2 Eric N. Hahn ${ }^{1,2}$, Timothy C. Germann ${ }^{2}$, Ramon Ravelo ${ }^{3,4}$, James E. Hammerberg ${ }^{3}$, Marc A.

3 Meyers ${ }^{1, \dagger}$

${ }^{1}$ Materials Science and Engineering Program, University of California San Diego, La Jolla, CA 92093, USA

${ }^{2}$ Theoretical Division, Los Alamos National Laboratory, Los Alamos, NM 94550, USA

${ }^{3} X$-Computational Physics Division, Los Alamos National Laboratory, Los Alamos, NM 94550, USA

${ }^{4}$ Physics Department and Materials Research Institute, University of Texas, El Paso, TX 79968, USA

† corresponding author: enhahn@eng.ucsd.edu

Abstract. Strain rate, temperature, and microstructure play a significant role in the mechanical response of materials. Using non-equilibrium molecular dynamics simulations, we characterize the ductile tensile failure of a model body-centered cubic metal, tantalum, over six orders of magnitude in strain rate. Molecular dynamics calculations combined with reported experimental measurements show power-law kinetic relationships that vary as a function of dominant defect mechanism and grain size. The maximum sustained tensile stress, or spall strength, increases with increasing strain rate, before ultimately saturating at ultra-high strain rates, i.e. those approaching or exceeding the Debye frequency. The upper limit of tensile strength can be well estimated by the cohesive energy, or the energy required to separate atoms from one another. At strain rates below the Debye frequency, the spall strength of nanocrystalline $\mathrm{Ta}$ is less than single crystalline tantalum. This occurs in part due to the decreased flow stress of the grain boundaries; stress concentrations at grain boundaries that arise due to compatibility requirements; and the growing fraction of grain-boundary atoms as grain size is decreased into the nanocrystalline regime. In the present cases, voids nucleate at defect structures present in the microstructure. The exact makeup and distribution of defects is controlled by the initial microstructure and the plastic deformation during both compression and expansion, where grain boundaries and grain orientation play critical roles.

\section{Keywords: tensile strength, spall, non-equilibrium molecular dynamics, tantalum}

30

\section{Introduction}

The tensile strength of metals is determined by the nucleation, growth, and coalescence of voids and/or cracks. At low strain rates, the applied traction generates an internal stress state that is relaxed by the introduction of these defects. As the strain rate is increased, stress-wave propagation becomes gradually more important and the stress state becomes increasingly nonuniform. Concomitantly, the competition between void nucleation, void growth, and wave propagation effects increases the complexity of the process. 
The high strain-rate regime is attained in uniaxial strain, a characteristic feature of shock

2 wave propagation, and in a geometry for which the lateral dimensions of the specimen are larger

3 than the pulse length. Tensile failure in this regime is commonly known as "spall" - a process of

4 physical damage evolution that is initiated by a rarefaction wave, or set of waves, whose amplitude

5 exceeds the local tensile strength of the material [1]. Upon exceeding the local tensile strength of

6 a material, ductile voids or brittle cracks nucleate and subsequently grow to relax the stress by

7 dislocation emission, twinning, or displace phase transformations [2]. In addition to the

8 microstructure, the duration and speed of the release dictates void concentration, sizes, and

9 distributions thereof [3]. Voids often grow via dynamic dislocation generation [4] and coalesce

10 into interconnected void volumes that may cause the material to undergo complete failure. If full

11 separation of the material is incomplete, the response is deemed incipient spall. The process of

12 void nucleation, growth, and coalescence is of critical interest in many fields due to the prevalence

13 of spall damage in engineering applications such as ballistic penetration as well as dynamic

14 fragmentation during hypervelocity impact events that occur in near orbit and outer space.

Dynamic fracture was first documented by Hopkinson [5] and has since seen a long history

16 of study using explosives, gas guns, flyer plates, and lasers [1,4,6-14] to induce tensile failure at

17 strain rates ranging from $10^{4}$ to $5 \times 10^{9} \mathrm{~s}^{-1}$. There is a strong experimental and theoretical foundation

18 that shows an increase in spall strength with increasing strain rate $[4,7,12,14]$. There is also

19 significant evidence that polycrystallinity decreases the spall strength as compared to single

20 crystals $[9,15,16]$. Christy et al. [17] performed experiments on polycrystalline copper and

21 observed clear differences in the frequency at which voids nucleate at grain/twin boundaries

22 compared to the grain interior for three different grain sizes - rationalized later by Meyers [18] and

23 Meyers and Zurek [19]. 
State of the art experimental studies use indirect measures (such as free surface velocimetry

$2[11,20]$ or Laue diffraction $[21,22])$ to infer the internal stress states of the material during dynamic

3 failure. Although highly useful, these experimental measurement techniques rely on accurate

4 equation of state (EOS) data (which prescribes the shock Hugoniot path) $[13,20,23,24]$ as well as

5 several bulk acoustic assumptions and simplifications that break down for realistic

6 microstructures, high strain rates, or large amounts of plasticity and/or damage evolution [25].

7 Several studies utilize post-mortem microscopy to identify damage, but it is often difficult to trace

8 damage back to a specific source [15] and the spall strength and damage field are not unique to

9 one another [26]. Other techniques are being developed, such as the use of high speed imaging

10 [27], that may address some of the issues enumerated here. Critically, spall models strongly rely

11 on empirical data $[25,28,29]$ for which many important quantities are often lacking.

Large scale molecular dynamics (MD) simulations have reached equivalent spatial and

13 temporal scales of laser-driven shock experiments and contain complete, time-resolved, atomic-

14 scale resolution of the relevant damage mechanisms [30-32]. Simulations allow for a well-

15 informed evaluation of spall strength at high strain rates and assist in our interpretation of

16 experimental results. The principal aim of this work is to identify void initiation conditions as a

17 function of defect structure and strain rate in order to establish macroscopic properties such as

18 cohesive strength, maximum sustained tensile load, and damage evolution. Under shock

19 compression it was shown that nanocrystalline metals exhibit ultrahigh strength [30] and here we

20 evaluate the strength of single and nanocrystalline metals under tensile release that often follows

21 the compressed state. For the present study, we focus on tantalum, a model body-centered cubic

22 (bcc) metal, for which an embedded atom model (EAM) potential well-suited for shock

23 simulations is applicable $[33,34]$. The chosen potential is able to reproduce important properties 
1 including the shock Hugoniot; properties of defects such as the gamma surface and energies of the

2 twinning/antitwinning paths; and the stress tensor resulting from uniaxial compressive strains [33].

3 The potential was also fit to the DFT cold curve of Ta up to 50\% volumetric expansion [33].

4 Details of the elastic properties under tensile stress are provided in the Supplemental Material.

5 There exist a number of important MD studies evaluating spall in model systems [35], $\mathrm{Cu}$ [16,36-

6 39], $\mathrm{Ni}$ [40], $\mathrm{Fe}$ [41], and Ta [42-45] plus several studies that evaluate the dynamics of void growth

7 and defect emission [46-49].

Critical findings of these prior studies identify that free surface velocimetry based

9 calculations underestimate the strain rate and can significantly overestimate the spall strength for

10 Taylor waves (shock waves with a dispersive tail) [37]. It is suggested that corrections to the bulk

11 sound speed and bulk density need to be taken into account to accurately represent the damaged

12 state; this was remarked on earlier by Chen et al. [25] when considering the validity of estimating

13 spall strength by traditional measurements. Multiple studies suggest that under strong shock

14 conditions the resulting defect structures created during compression may overshadow pre-existing

15 defects and thus the initial microstructure or crystalline orientation plays a limited role during spall

16 failure $[36,42,50]$. Other studies show that the character of defects present in the original

17 microstructure, such as specific grain boundaries or grain sizes, play a significant role in the

18 observed spall strength of metals [9,15]; Mackenchery et al. [16] explicitly show that an increased

19 twin presence in certain nanocrystalline grain sizes influences the peak tensile stress at which voids

20 nucleate at grain boundaries for face-centered cubic (fcc) $\mathrm{Cu}$.

21 It has been demonstrated that the high strain rates produced through laser-shock techniques

22 decrease the mean spacing between nucleated voids [3]. Voids continue to nucleate throughout the

23 nucleation-growth-coalescence process [44] and the damage evolution and sustained tensile 
1 strength exhibits critical behavior relating to the growth of sufficiently large voids [42]. The

2 growth of voids is kinetically limited by the emission of defects such as twins, dislocations, and

3 plastic flow that depend on the strain rate, size of the void, and the complex stress state around

4 them [48].

Ultimately, we aim to describe the spall strength, $\sigma_{\mathrm{sp}}$, of tantalum as a function of microstructure, strain rate $(\dot{\varepsilon})$, and temperature $(T)$. We can enumerate a basic formalism that the 7 spall strength is a function of these variables:

$$
\sigma_{s p}=f(Z, s, \dot{\varepsilon}, T)<\sigma_{\max }(Z, s)
$$

9 where $\mathrm{Z}$ defines the material composition and phases; $\boldsymbol{s}$ is a set of microstructure descriptors, 10 including grain size statistics (mean size, distributions of size and/or shape, etc.), texture, grain 11 boundary and triple junction character, and so forth. The variables in Eq. (1) are intrinsically 12 coupled to one another. For instance, strain rate influences the type and quantity of defects that 13 form, this in turn dictates increases in the local temperature from the heat generated by plastic 14 work as defects move through the lattice, and the resulting temperature adjusts the intrinsic barriers 15 to further dislocation motion. This interconnected series of processes is especially crucial during 16 spallation events where the location of spall failure is highly history dependent. It has been 17 established that void nucleation depends principally on the rate, which stems from the role of time 18 on the connected dislocation processes such as thermally activated or viscous-drag limited motion.

19 Relatedly, increasing the strain rate raises the spall strength of the material, a process that relates

20 the competition between void nucleation and void growth [14,51]. Both processes are intimately

21 tied to dislocations, both nucleation and the subsequent motion thereof, which are effected by the 
1 plastic strain rate according to the classical Orowan [52] equation. For strain-rate experiments

2 greater than $10^{4} \mathrm{~s}^{-1}$, the spall strength is typically expressed with a power law relationship $[12,14]$

$$
\sigma_{s p}=\sigma_{0}(\dot{\varepsilon})^{m_{1}} .
$$

3 We will expand on these general relationships throughout the manuscript and use them as an

4 underlying foundation upon which to build.

\section{Simulation Methods}

Multiple non-equilibrium molecular dynamics simulation methods are employed to evaluate dynamic tensile failure in the strain-rate space of $10^{7} \mathrm{~s}^{-1}-10^{13} \mathrm{~s}^{-1}$. These results will be benchmarked against density functional theory (DFT) predictions and EOS calculations. Three methodologies are employed, illustrated graphically in Fig. 1, with characteristic snapshots at time $0,30 \mathrm{ps}$, and $60 \mathrm{ps}$. Each simulation archetype offers specific advantages and disadvantages, as we describe next.

\subsection{Flyer Plate and Target}

The collision between a flyer plate and a target (Fig. 1(a)) provides supported square wave stress profiles, where failure is well defined at a spall plane based on geometric relationships and acoustic approximations for wave speeds [37]. Both the flyer and the target are assigned velocities in the shock direction such that the spall plane remains close to constant position with nearly zero center of mass velocity [37]. The flyer plate itself is often not analyzed and results in wasted computational resources. Lower strain rates can only be achieved by extending the lengths of the flyer plate and target, allowing the two resulting rarefaction fans more time to spread before they collide and pull the target into tension. The computational cost thus grows quadradically with 
1 (inverse) strain rate, as a 10x slower strain rate requires 10x more atoms for a 10x longer simulated

2 time, a 100x more expensive computation.

More general loading conditions, such as those arising from high explosive (HE), laser, or magnetic drives, can be modeled by an arbitrary time-dependent piston velocity (Fig. 1(b)). The controlled acceleration and deceleration profiles can reproduce the stress profile reminiscent of those achieved during laser shock or HE experiments, with uniaxial strain followed by a dispersive tail $[53,54]$. The strength, duration, and shape of the imposed piston velocity (which translates directly to the particle velocity, $U_{p}$ ) will dictate the prescribed shock volume and strain rate [55]. For the present simulations, the piston is linearly ramped to $U_{p}=750 \mathrm{~m} / \mathrm{s}$ over 5 picoseconds (ps). A resultant shock wave is formed within 15 ps (Supplemental Material), and the sharpness of the shock front can be seen in Fig. 1. The piston velocity is maintained at this magnitude for 20 ps and then undergoes a linear deceleration to stationary over 20 ps - this implementation creates an unsupported shock wave. In order to control the strain rate it is necessary to extend the system size and simulation time to decrease the strain rate towards those achieved in experiments. This requires a precise description of non-linear dissipation mechanisms that contribute to the decay of the shock as it travels and large dimensions in the shock direction to achieve steady state and slower strain rates, with the same quadratic computational cost as the flyer plate and target approach, but with approximately two thirds of the total atoms. While both the flyer-target and controlled piston non-equilibrium molecular dynamics (NEMD) simulations are more expensive, they provide the correct density of defects which develop within nanoseconds in relevant laser shock experiments. As it turns out, the density of defects plays an important role in spall kinetics. 
The quasi-isentropic (QI) technique shown in Fig. 1(c) allows for homogeneous compression

2 and expansion of the material at a constant and well-defined strain rate across the entire sample.

3 Within this method, failure can occur at any point in the simulated volume, where the cohesive

4 strength is weakest. This is especially important for nanocrystalline specimens where other

5 methods may apply the maximum tensile stress to a limited subset of grain boundaries within the

6 spall plane or volume. This method also allows for sampling of much lower strain rates compared

7 with those generated in typical NEMD shock simulations of spall, and at a comparable

8 computational cost. For the present QI simulations, we employ a fully periodic cubic system with

9 side lengths of $66 \mathrm{~nm}$ consisting of 16 million atoms. The primary limitation of the system size is

10 the communication between growing voids [1,10]. The system undergoes an initial compression

11 to 0.17 strain at a strain rate of $10^{9} \mathrm{~s}^{-1}$ remapping the atomic positions in the loading direction every

12 time step (1 fs) after which the stress state is held constant for $100 \mathrm{ps.} \mathrm{The} \mathrm{"hold"} \mathrm{allows} \mathrm{for} \mathrm{defect}$

13 relaxations - analogous to relaxations that occur behind the shock front.

15 approximation (with the response of tantalum measured by [12]) for the NEMD piston shock,

$16 \dot{\varepsilon}=27.34 \cdot 10^{36}\left(\sigma_{z z}{ }^{4}\right)=2.5 \cdot 10^{8} s^{-1}$, and an acoustic approximation using a longitudinal sound speed

17 of $5400 \mathrm{~m} / \mathrm{s}, \quad \dot{\varepsilon}=U_{p} /\left(t \cdot C_{L}\right)=2.8 \cdot 10^{10} \mathrm{~s}^{-1}$. It is important to note that adjusting the compressive

18 strain rate will result in different defect densities, which, in turn, will influence the tensile response.

19 A promising avenue for a future study would be to detail this dependence. The strain of 0.17 was

20 also chosen to match the peak shock pressure in the NEMD simulations. The peak longitudinal

21 stress for [001] crystals is $55.5 \mathrm{GPa}$ and the nanocrystalline system sees $54.5 \mathrm{GPa}$. The initial QI

22 compression is followed by QI tension (at varying strain rates) until failure - this sequence is meant

23 to be comparable to shock compression or a fast ramp to a specified shock pressure followed by 
1 isentropic release. The peak shock pressure (corresponding to $U_{p}=750 \mathrm{~m} / \mathrm{s}$ ) is $56.5 \mathrm{GPa}$ for the

2 [001] single crystal and $52 \mathrm{GPa}$ for the nanocrystal. For comparison, the QI strained systems reach

3 peak compression pressures of 55.5 and $54.5 \mathrm{GPa}$ for the [001] and nanocrystalline systems. The

4 dislocation density (comprised primarily of twinning dislocations) of single crystalline samples at

5 peak compression is $6.4 \times 10^{12} \mathrm{~cm}^{-2}$ and $6.9 \times 10^{12} \mathrm{~cm}^{-2}$ for NEMD shock and QI methods

6 respectively. The close agreement between the dislocation densities obtained in both methods

7 shows that the strain rate chosen for the QI method produces very similar structural states under

8 compression as those seen in the NEMD shock (piston) simulations for the same compressive

9 strains. It is worth noting that shocked systems release isentropically and QI simulations are

10 faithful to this process; varying tensile strain rates are representative of systems with much larger

11 "target" lengths where the imposed compressive wave has a greater period of time over which to

12 disperse, resulting in lower tensile strain rates.

13 Since the required simulation volume is independent of strain rate (with the only

14 requirement being that it is large enough to contain a representative defect and void

15 microstructure), the computational cost is only linear in tensile strain rate, allowing much lower

16 strain rates to be accessed.

\subsection{System initialization, integration, and visualization}

Nanocrystalline samples were generated using SPaSM [59] and simulations were run using

19 either SPaSM [60,61] or LAMMPS [62]. The flyer-target and piston configurations employ

20 periodic boundary conditions transverse to the shock direction (with lateral dimensions of $\sim 50 \mathrm{~nm}$ )

21 and allow for unconstrained expansion of the free surface in the shock direction. The flyer and

22 target are $75 \mathrm{~nm}$ and $150 \mathrm{~nm}$ long respectively; the piston system is $150 \mathrm{~nm}$ in length. Both systems

23 are shocked along the [001] single crystal orientation. The [001] orientation was selected to best 
1 relate the present simulations to prior experimental results in literature. The resulting system sizes

2 are 30 million atoms for the flyer-target and 20 million atoms for the piston system. We employ 3 an EAM potential (Ta1) that is well suited for high pressure studies of tantalum $[33,34,63]$ at

4 relatively low computational cost [64]. The second order elastic moduli of both Ta potentials (Ta1

5 and Ta2) developed by Ravelo et al. [33] were evaluated and show good stability up to 30 GPa in

6 tension (Supplemental Material). We visualize our results using OVITO [65] with its integrated

7 adaptive common neighbor analysis, coordination analysis, dislocation extraction algorithm

8 (DXA) [66] and surface meshing tools [67]. The nanocrystalline samples evaluated here have a

9 mean grain size of $21 \mathrm{~nm}$ and are thermally annealed at $0.7 \mathrm{~T}_{\mathrm{m}}$ for $0.5 \mathrm{~ns}$. Both the single and

10 nanocrystals are thermalized to $300 \mathrm{~K}$ prior to loading.

\section{3. Results and Analysis}

The flyer-target and piston shock methodologies both provide an opportunity to evaluate

14 the free surface velocimetry compared to the internal stress state at the spall plane; however, at

15 present, we select to focus solely on measurements and calculations taken within the specific spall

16 volume. A comparison of these two methods, highlighting differences in shock stress, $\sigma_{\mathrm{zz}}$, is shown

17 in Fig. 2(a-b). For stress calculations we bin along the shock direction taking a virial stress

18 definition [68]:

$$
\sigma_{\alpha \beta}=-\frac{1}{\Omega_{a}}\left[m v_{\alpha} v_{\beta}+\frac{1}{2} \sum_{n=1}^{N} r_{1 \alpha} F_{1 \beta}+r_{2 \alpha} F_{2 \beta}\right] .
$$

$19 \Omega_{\mathrm{a}}$ is the atomic volume; $\mathrm{m}$ is the mass; $\mathrm{v}$ is the velocity; $\alpha$ and $\beta$ mark the Cartesian components;

$20 \mathrm{~N}$ is the number of paired neighbors looped over by the variable $n ; F_{1}$ and $F_{2}$ are the forces on a

21 pair of atoms; and $r_{1}$ and $r_{2}$ are the positions of the atoms in the pairwise interaction. The kinetic 22 energy term accounts for binned center-of-mass translational motion. The minimum of the 
1 longitudinal component of the stress tensor evaluated for each bin ( 50,000 atoms) in time is

2 defined to be maximum sustained tensile strength. Figure 2(c,d) illustrates the evolving density

3 and Fig. 2(e,f) presents the temperature evolution. Features of these figures will be extended upon

4 in subsequent sections. For the piston and flyer methodologies, the instantaneous true strain rate

5 at a given Eulerian position along the shock direction (z) can be expressed from the position

6 dependent density at subsequent time steps,

$$
\left.\dot{\varepsilon} \equiv \frac{1}{\rho} \frac{\partial \rho}{\partial t}\right|_{z}
$$

7 The strain rate can also be represented as spatial derivatives of the particle velocity and density 8 evaluated at a single point in time:

$$
\dot{\varepsilon}=\left.\left(\frac{\delta u_{z}}{\delta z}+\frac{u_{z}}{\rho} \frac{\delta \rho}{\delta z}\right)\right|_{t}
$$

9 The form of Equation 5 is used in order to evaluate the longitudinal strain-rate at a specific point 10 in time.

11 In all of the present simulations, we measure the stress in the shock direction, $\sigma_{z z}$, the strain $12(\varepsilon)$, temperature $(\mathrm{T})$, hydrostatic pressure $(\mathrm{P})$, and deviatoric shear stress $(\tau)$. The hydrostatic 13 pressure is calculated as

$$
P=\left(\sigma_{x x}+\sigma_{y y}+\sigma_{z z}\right) / 3
$$

14 Lateral stresses $\left(\sigma_{\mathrm{xx}}\right.$ and $\left.\sigma_{\mathrm{yy}}\right)$, roughly equivalent to one another, are used in the calculation of 15 deviatoric shear stress [32]:

$$
\tau=\frac{1}{2}\left(\sigma_{z z}-\frac{1}{2}\left(\sigma_{x x}+\sigma_{y y}\right)\right) .
$$

Temperature is calculated as: 


$$
T=\frac{1}{n} \sum_{n=1}^{n} \frac{m}{3 k_{b} N_{A}}\left(v_{n, x}{ }^{2}+v_{n, y}{ }^{2}+v_{n, z}{ }^{2}\right) .
$$

1 The mass of tantalum $m=180.95 \mathrm{u}, k_{b}$ is Boltzmann's constant, $N_{A}$ is Avogadro's number, and the

2 components of the velocity vector are $v_{x}, v_{y}$, and $v_{z}$. During spall failure we limitedly employ a

3 two-dimensional definition of temperature $\left(T \propto\left(\mathrm{vx}^{2}+\mathrm{Vy}^{2}\right) / 2 \mathrm{k}\right)$ in order to negate the need to

4 subtract the center of mass translational velocity from the longitudinal velocity component, $\mathrm{v}_{\mathrm{z}}$.

5 This is critical when evaluating the temperature evolving around a three dimensional defect, such

6 as a void, where an applicable center-of-mass translational velocity for a group of atoms may not

7 be representative of the translational velocity at a particular point in space.

\subsection{Spall Strength as a Function of Strain Rate}

Fig. 3 shows spall strength as a function of strain rate, including experimental and simulation results from literature. The plot confirms the pertinence of using molecular dynamics to explore the extreme strain-rate regime. Points are colored according to their grain size; black points represent [001] single crystals, blue/purple represent polycrystals, and red points represent nanocrystals. There is a clear difference in slope between the power law curves fit to mono, poly, and nanocrystalline data (Supplemental Material) and key aspects of failure within single crystalline and nanocrystalline samples are detailed in Sections 3.2 and 3.3 respectively. Temperature plays an important role in the failure process and the effect is described in Section 3.4. The theoretical cohesive stress $\sigma_{\max }$, reached at strain rates exceeding the Debye frequency is similar for varying grain sizes, but does show a residual effect of the orientation/grain size. We discuss the theoretical cohesive stress in section 3.5.

Key results at tensile strain rates spanning $10^{7}-10^{13} \mathrm{~s}^{-1}$ are located in Table I and Table II. In addition to the simulation method and tensile strain rate (which is directly imposed for QI, and indirectly for the flyer plate and piston methods), the peak tensile stress in the shock direction $\left(\sigma_{\mathrm{zz}}\right)$ 
1 and the corresponding hydrostatic pressure $(\mathrm{P})$, deviatoric shear stress $(\tau)$, strain $(\varepsilon)$, temperature

$2(\mathrm{~T})$ are given.

\section{2. [001] Single Crystalline Tantalum}

4

The peak tensile stress (i.e., the spall strength) as a function of strain rate is graphically shown in Fig. 4 for single crystals. Insets within the figure demonstrate which deformation mechanism is dominant at a given strain rate. Power law trends depicted as linear fits in the loglog plot illustrate that the kinetics of spall is deformation mechanism dependent. At strain rates greater than $5 \times 10^{10} \mathrm{~s}^{-1}$ the mechanism governing tensile strength again changes. At elevated strain rates the material has significantly less time to respond to the applied stress and failure occurs primarily by de-cohesion of atoms (Fig. 4 inset). This primarily occurs in areas of reduced density, such as at twins that have excess volume (and energy) within the boundary layer [61]. A complete analysis of extreme strain rates is left for future study - the possibility of phase change at tensile strains in excess of 0.25 remains an open question[69] and exceeds the bounds the potential was fit to. The following tensile strain-rate regimes are delineated by their dominant deformation mechanism: dislocations, $\dot{\varepsilon}<10^{9} \mathrm{~s}^{-1}$; twinning, $10^{9} \mathrm{~s}^{-1}<\dot{\varepsilon}<5 \times 10^{10} \mathrm{~s}^{-1}$; de-cohesion, $\dot{\varepsilon}>5 \times 10^{10}$ $\mathrm{s}^{-1}$. The curved vertical line on the right hand side of the figure depicts the Debye frequency as a function of tension, which serves as a good estimate for where the material should exhibit its ultimate tensile strength [35]. The upper limit of tensile strength is indicated by a horizontal dashed line and a discussion of its value will follow in the final section.

Single crystal simulations show that dynamic spall proceeds via the nucleation of several distributed voids at defects that are generated during compression as well as in tension. A time series of the flyer-target and piston simulations are shown in Fig. 5 (refer to full videos in the supplemental material). The snapshots correspond to the time of maximum tensile stress $\left(t_{\max }\right)$ and 
16,4 , and 2 ps before $t_{\max }$ as well as 2 and 4 ps afterwards. The local longitudinal stress state is

2 represented by the atom's color, blue corresponding to compressive stress and orange to tensile

3 stress. Defects are marked in dark green and void surfaces in dark red. For the flyer plate system,

$4 t_{\max }=56 \mathrm{ps}$, and for the piston-driven system, $\mathrm{t}_{\max }=61 \mathrm{ps}$. The difference in timing is in large part

5 due to differences in pulse width/shape and the paths through which the waves travel. The spall

6 plane for the flyer-driven system does not lie precisely at the geometrically prescribed location,

7 but instead deviates proportionally to the change of the shock velocity in the relative amounts of

8 uncompressed, compressed, and expanded media the wave travels through, in addition to non-

9 linear dispersion effects that cause the initial wave to broaden.

For both loading conditions, we observe that voids in single crystalline tantalum nucleate

11 at twin-twin intersections as was shown previously for strain rates near $10^{10} \mathrm{~s}^{-1}$ [55]. QI simulations

12 at comparable strain rates show similar behavior with voids nucleating primarily at twin

13 intersections where local stress concentrations arise (Fig. 6). Additionally, we identify

14 interconnected symmetric and sheared $\Sigma 3$ twin boundaries [70] (inset in Fig. 6) which indicates

15 that deformation twinning in tantalum favors dissociated $1 / 12<111>$ partials traveling along

16 adjacent $\{211\}$ planes as opposed to $1 / 6<111>$ dislocation glide [71]. The fact that all three

17 simulation types (at comparable strain rates) show similar defect and void mechanisms gives

18 credence to comparisons between the methods.

Figure 7 returns our focus to the piston NEMD simulation illustrating the evolution of twins

20 and voids, as well as their relationship to the local longitudinal stress and temperature. Snapshots

21 every 2 ps between 56 and 64 ps are shown highlighting the early stages of void nucleation, growth,

22 and rapid coalescence due to the proximity of the voids at high strain rates and high twin density.

23 Three visualization schemes are shown. The top row gives atoms identified by their potential 
1 energy, which serves to highlight twins and void surfaces. The middle row colors atoms according

2 to the local stress in the shock direction. This allows for the identification of local stress

3 concentrations due to compatibility requirements within the twinned microstructure and also

4 shows the rapid release and relaxation of tensile stress during the growth of voids. The maximum

5 tensile stress of $19.1 \mathrm{GPa}$ occurs at $61 \mathrm{ps}, 5 \mathrm{ps}$ after the first void nucleates at $15.9 \mathrm{GPa}$. A growing

6 void's ability to relieve stress is proportional to its size. This relationship is a ramification of the

7 reduced stress required to nucleate dislocations for larger voids [48] in addition to the greater

8 number of dislocation nucleation sites (which is proportional to the surface area of the void) and

9 increased volume that nucleated dislocations can expand into [72]. It is not until sufficient void

10 growth that the stress relaxation rate equals and surpasses the stress rate. Lower strain rates, and

11 thus lower stress rates, require less cumulative damage to relax the structure. The bottom row

12 presents the local temperature, which increases drastically during void growth due to the

13 irreversible visco-plastic work. The effect of increased temperature will be discussed in greater

14 detail later in the manuscript.

15 The propensity for deformation to occur via twinning or full dislocations depends on the

16 strain rate (as well as grain size and orientation [33,34,73]). At lower strain rates, where

17 dislocations are more favorable than twins, voids nucleate at individual dislocations or at nodes

18 between them. This is visualized in Fig. 8 for QI tension at a strain rate of $10^{7} \mathrm{~s}^{-1}$. The system was

19 tracked for porosity and dislocation density during the principal void nucleation event and

20 subsequent void growth. A series of snapshots in time are presented in Fig. 8 alongside the

21 evolution of void volume and dislocation density.

22 Our simulation corroborates the findings by Strachan et al. [42] that implicate a critical

23 void volume prior to failure. In our simulation this occurs by the dynamic growth of a single critical 
1 void. Furthermore, previous calculations for void growth [48] are based upon dislocations

2 "carrying away" volume from the growing void [2]. The volume transported by a dislocation can

3 be considered, as a first approximation, as the length of the dislocation times a core area. In tension,

4 the dislocation core is larger than at equilibrium (which is approximately the square of the Burgers

5 vector, $b^{2}$ ), measuring $10.7 \AA^{2}$ at $25.3 \mathrm{~ns}$ (measured presently using a representative volume mesh

6 [67] of defective atoms belonging to a dislocation core divided by the dislocation length). Figure

78 presents a calculation of dislocation density times the system volume (to give dislocation line

8 length) multiplied by the average core area at each step. The analytical calculation begins at the

9 critical void nucleation time, $25.3 \mathrm{~ns}$. This time also corresponds to the minimum dislocation

10 density as a consequence of dislocation annihilation in part due to the Bauschinger effect.

11 Figure 9 shows a magnified snapshot of the previous simulation at $24.5 \mathrm{~ns}$; shear loops

12 (attached to the void) and prismatic loops (detached) are identified. First, shear loops are emitted

13 from the void surface. The edge component of the shear loops evolves, leaving behind the lateral

14 sides, which have screw character. These can cross-slip into different $\{110\}$ planes which intersect

15 along the same [111]. Thus a "lasso-action" takes place, which creates, on closing itself, a

16 prismatic loop. This "lasso" action mechanism was first observed in void growth in tantalum [48]

17 and later confirmed in deformation under indentation at relatively high strain rates [74].

$18 \quad 3.3$. Nanocrystalline Tantalum

19 The spall strength of nanocrystalline tantalum $(\mathrm{d}=21 \mathrm{~nm})$ as a function of strain rate is

20 also given in Fig. 4. Results for these simulations are provided in Table II. Unlike single crystals,

21 the nanocrystals demonstrate a single power law relationship over the strain rates of $10^{8}-10^{13} \mathrm{~s}^{-1}$,

22 suggesting a single dominant mechanism. 
Figure 10 shows snapshots of quasi-isentropic expansion at strain rates of $10^{8}-10^{10} \mathrm{~s}^{-1}$. In

2 contrast to simulations of tensile failure by Tang et al. [75] and Belak [3], and in agreement with

3 Rudd [45], we observe that grain-boundary nucleation of voids occurs in concert with limited

4 dislocation and twin nucleation. The first column of Fig. 10 displays a sliced image that is colored

5 according to longitudinal stresses averaged over $0.5 \mathrm{~nm}^{3}$ spherical volumes. The second column

6 depicts atoms filtered by adaptive common neighbor analysis [76] showing only defective atoms,

7 this method determines an optimal cutoff radius automatically for each individual atom. The atoms

8 are colored by a "defect coordination" allowing for the visualization of grain boundaries, full

9 dislocations, and twin surfaces. The third column uses a potential energy filter (with a cutoff of -

$10 \quad 7.2 \mathrm{eV}$ ) that only shows atoms on the surface of voids with higher average energy than the

11 surrounding crystal, grain boundaries, or crystalline defects. A selective coordination coloring

12 scheme is employed where increasing void volume corresponds to voids of blue, turquoise, yellow,

13 orange and green coloring. Spherical voids would be constant in color; the degree of color change

14 along the void surface is a qualitative measure of void eccentricity.

Figure 11 gives a side-by-side comparison between single and nanocrystalline samples, for

16 piston loading to shock pressures of $56.5 \mathrm{GPa}$ and $52 \mathrm{GPa}$ in single crystal and nanocrystal

17 respectively. The corresponding strain rate under release is $1.65 \times 10^{10} \mathrm{~s}^{-1}$ in the single crystal and

$187.8 \times 10^{9} \mathrm{~s}^{-1}$ in the nanocrystal. The top frames give the complete atomic pictures, while the bottom

19 frames show surface meshes of the evolving voids. Within the single crystal, voids appear to

20 nucleate randomly through the spall volume. In the nanocrystalline sample, voids nucleate along

21 the grain boundaries. This process is also apparent by viewing the $x-t-\rho$ diagrams of Fig. 2. A

22 qualitative analysis suggests that specific grain boundary misorientations do not play a large role,

23 but that boundary orientation relative to the loading direction is important - boundaries that are 
1 perpendicular to the loading direction fail preferentially. As voids nucleate and grow along the

2 grain boundaries the frequency of coalescence is increased due to their proximity to one another.

3 At strain rates lower than $10^{11} \mathrm{~s}^{-1}$ the spall strength of nanocrystalline Ta is below single crystalline

4 Ta. This occurs in part due to the decreased flow stress of the grain boundaries; stress

5 concentrations at grain boundaries that arise due to compatibility; and the growing fraction of

6 grain-boundary atoms as grain size is decreased into the nanocrystalline regime. At higher strain

7 rates, the orientation of the crystal (or group of crystals in the case of a nanocrystal) will matter to

8 a much greater extent as the orientation will significantly influence de-cohesion.

$9 \quad$ 3.4. The Role of Temperature During and After Spall

10 Temperature plays an important role in the softening of tantalum prior to, and during spall.

11 As mentioned in the Methodology Section and by Ravelo et al. [56], QI simulations maintain

12 constant temperature excluding the work and heat generated by plasticity. Thus, for a fixed strain

13 rate, it is possible to vary the "initial" temperature and maintain a level of control over the eventual

14 temperature at which spall occurs. For these select "temperature-varied" simulations, a procedure

15 consistent with the QI simulations presented above is performed with the following modifications:

16 a 17\% uniaxially compressed state was generated, as before, by QI compression starting at room

17 temperature using a compressive strain rate of $10^{9} \mathrm{~s}^{-1}$. The system was then equilibrated using NPT

18 to a target temperature for 100 ps while maintaining the stresses of the $17 \%$ compressed state -

19 this differs from the previous simulations which use an NVE ensemble to allow for defect

20 relaxation over 100 ps. Volume expansion and dislocation annealing is expected to occur during

21 this period; the goal is to create states with comparable defect structures while varying the initial

22 temperature in order to isolate the effect of temperature on the spall strength. 
We select a tensile strain rate of $10^{9} \mathrm{~s}^{-1}$ to evaluate due to its correspondence to laser driven

2 spall experiments [11]. Figure 12 presents the measured spall strength as a function of increasing

3 temperature. The temperature is evaluated at the maximum spall strength and thus includes some

4 of the heat generated through the visco-plastic work that is associated with the spall. Spall strength

5 decreases with increasing temperature for both solid and liquid Ta.

6 We first focus on the spall strength of liquid Ta for simplicity. The cavitation strength of

7 liquids has particular relevance to laser-driven systems where large temperatures are generated

8 through laser-matter interactions. They are also relevant to strong shock conditions where melting

9 may occur under compression or during release. We identify a dependence of spall strength on

$10 \sim 1 / \mathrm{T}$, agreeing with previous simulations of spall in liquid $\mathrm{Cu}$ [36] and other metals [77]. The exact

11 relationship for cavitation strength identified is

12

$$
\left.\sigma_{c a v}^{l i q}\right|_{10^{9} s^{-1}}=\mathrm{A}_{\text {liq }} / T^{1.064},
$$

13 where $\mathrm{A}_{\mathrm{liq}}=36628 \mathrm{GPa} * \mathrm{~K}$. Ashitkov et al. [11] provide an experimental measurement of the spall 14 strength (cavitation strength) of liquid tantalum as melted under laser irradiation, at an estimated 15 strain rate of $1.5 \times 10^{9} \mathrm{~s}^{-1}$ and a temperature of $\sim 5000 \mathrm{~K}$. As seen in Fig. 12 (solid red circle), this 16 single data point for molten Ta agrees well with the present simulations. There exists a discontinuous transition between the solid and liquid response; this indicates 18 that plasticity continues to play an important role in failure up until the point of melting. In the 19 solid regime, the decrease of the spall strength with temperature can be described with a power 20 law relationship:

$$
\left.\sigma_{s p}^{s}\right|_{10^{9} s^{-1}}=\sigma_{0}^{s}-\mathrm{A}_{s} T^{1.646} .
$$


1 Here, the intrinsic spall strength, $\sigma_{0}^{s}$, at a reference strain rate of $10^{9} \mathrm{~s}^{-1}$ and $0 \mathrm{~K}$ is equal to 14.33

$2 \mathrm{GPa}$. The constant in front of the inverse temperature dependence, $\mathrm{A}_{s}=9.8 \times 10^{-6} \mathrm{GPa} / \mathrm{K}$. A similar

3 relationship form was observed for copper [36]. The origin of the equation is akin to the Simon

4 equation which describes the temperature at which a material melts at a given pressure. In general

5 it describes the softening of a material by a power law by making several thermodynamic 6 assumptions [78].

7 The local temperature increase can be quite substantial, as shown in Fig. 2. In the spalled

8 region the average temperature is often greater than $1500 \mathrm{~K}$, or roughly half of the ambient melting

9 temperature. Figure 13 shows snapshots in time of a spalled system over a 40 ps timespan,

10 demonstrating the localization and increase of temperature during void growth and the persistence

11 of high temperature during coalescence and failure that results in long, ductile, and molten

12 ligaments connecting the material together. Such ductile material linkages have been seen in other

13 studies $[79,80]$. The high melting point of tantalum aids in its sustained ability to resist failure.

\subsection{Theoretical Predictions of Tensile Strength}

The theoretical maximum cohesive stress can be investigated through an analysis of the material's equation of state [25], specifically the cold-pressure curve [22]. At their core, interatomic potentials provide the energy of an atom, U, with respect to its neighbors' positions, $\mathrm{r}_{\mathrm{ij}}$. The hydrostatic cold pressure is related to the volume an atom occupies, $\mathrm{V}_{\mathrm{i}}$, by $\mathrm{P}=\mathrm{dU} / \mathrm{dV}$. A minimum value of pressure, corresponding to a maximum "negative" pressure, can be obtained by finding the critical zero value of the derivative of pressure with respect to volume: $0=\mathrm{dP} / \mathrm{dV}$. This process will yield a critical volume that can be input into the expression for pressure to yield a measure of the theoretical cohesive stress. Grady [22] used a simplified volumetric Morse potential in order to obtain an analytical solution: 


$$
P=\left(\frac{B_{0} U_{c o h}}{8 v_{0}}\right)^{1 / 2} .
$$

7 radial form $\left(U=D_{0}\left[e^{-2 \alpha\left(r-r_{0}\right)}-2 e^{-\alpha\left(r-r_{0}\right)}\right]\right.$, and density functional theory (DFT) calculations by

Using values from Rose et al. [25] $\left(\mathrm{B}_{0}=194.2 \mathrm{GPa}, \mathrm{U}_{\text {coh }}=8.089 \mathrm{eV}\right.$, and $\left.\mathrm{v}_{0}=0.202 \mathrm{~nm}^{3}\right)$, equation 11 predicts an ultimate tensile strength for tantalum of $39.4 \mathrm{GPa}$, The procedure above can be completed for interatomic potentials ("molecular dynamics" equations of state) and density functional theory calculations in order to provide more accurate predictions of the theoretical strength. This is shown in Fig. 14, which compares the Ta1 EAM potential utilized here with the closely related Ta2 potential [5], an EOS derived by Rose et al. [25], a fit of a Morse potential in Ravelo et al. [5]. A comparison of other potentials is given in the supplemental material. The prediction for the radial Morse potential is $30.5 \mathrm{GPa}$, Ta1 is $31.6 \mathrm{GPa}$ and for DFT is $33.4 \mathrm{GPa}$, which compare remarkably well with 31-34 GPa strengths measured using NEMD simulations (Tables I and II, Figs. 8 and 13). The fact that the radial Morse potential has improved agreement as compared to the estimate stemming from the volumetric Morse form is due to differences in the coefficient " 8 ". Based on the present study, a coefficient of 13.4 inside the radical of Equation 11 provides a better estimate of the ultimate tensile strength.

\subsection{Discussion}

The quasi-isentropic simulations are set up such that the imposed uniaxial strain rate is constant. However, the resulting rates at which the stress components evolve are not constant. The rate of change of hydrostatic pressure with time can be estimated according to the pressure-volume relationship. By taking the first derivative of the U-V curve we obtain a P-V relationship, also shown in Fig. 14. Where the P-V curve is linear, the stress rate can be directly related to the strain rate, i.e., in the elastic regime. With increasing tensile strain, the rate at which (negative) pressure 
1 increases per strain increment decreases - this is a ramification of the convexity. After void

2 nucleation, the rate of pressure change will decrease due to stress relaxation of the growing voids,

3 as well as the nucleation of additional voids. The size and number of voids will control the rate of

4 plastic relaxation, which is tied to the dislocation density around the growing voids. The plastic

5 relaxation rate will thus be a function of the dislocation generation and dislocation motion. After

6 time, the relaxation rate and the rate of tensile loading will be equivalent; at this point the material

7 has reached its maximum supported tensile stress, i.e., the spall strength. The material then returns

8 to zero stress as voids continue to grow and coalesce. If the stress returns to zero before the

9 coalescence of voids leads to a complete spall surface, the spall is incipient. This is especially the

10 case for short pulse durations. Often the rate of pressure change goes to zero and reverses sign as

11 the material around the voids is recompressed.

12 One difference between decaying and supported waves is worth further discussion here.

13 For a supported wave the resulting voided volume must be larger to completely relax the stress of

14 a larger volume of stressed material. This is simply because the tensile loading rate will be applied

15 for a greater period of time - relaxation must also continue for a longer duration. A similar logic

16 can be applied to higher strength shocks. Such shocks contain greater kinetic energy (and thus

17 momentum) that must be compensated for. For events where the unloading rate is the same, but

18 the shock strength is different, the material will continue undergoing a tensile driving force that is

19 countered by the relaxation rate. The tensile pulse does not stop when the material begins to

20 undergo spall; instead, a myriad of waves are emitted from growing void surfaces that counteract

21 the loading pulse. The sum of these waves may negate the tension, but the tensile wave still "exists"

22 even though it is masked by the other waves. From this standpoint, a larger extent of damage can

23 be expected for higher shock strengths or shocks of longer duration, but the spall strength of the 
1 material will be equivalent. This has been discussed previously in terms of square vs. triangular

2 waves by Tonks et al. [82] and Koller et al.[83]. Square waves continue to provide a driving

3 momentum to spall "scab", or separated layer, for a greater duration than unsupported triangular

4 waves. However, the temperature rise at the shock front (and the temperature increase due to

5 visco-plastic deformation) has the ability to alter the spall strength as a result of thermal softening.

6 It is to be expected that stronger shocks or longer shock pulses will cause a greater degree of

7 plasticity and thus a larger decrease in spall strength if the compression wave is reflected and

8 returns to the compressive defect field. One can imagine that for lower strain rates the plastic

9 relaxation rate will be much larger than the respective tensile loading rate, and that the strain

10 between nucleation and the maximum stress will be substantially reduced.

11

\section{Conclusions}

The ultimate strength of materials is an important property and represents the stress at which the interatomic forces can no longer sustain the cohesion of the structure. In quasi-static tension the value is never reached or even approached as a variety of mechanisms conspire to lower the maximum sustained tensile stress. However, as the strain rate is increased, this value rises and in the shock wave regime the stress state (uniaxial strain) and the kinetics of void/crack nucleation, growth, and coalescence are such that the ultimate stress is reached. With respect to Eq. 1, the roles of temperature, microstructure, and strain rate were evaluated for tensile failure bcc tantalum.

The sustained stress at failure is dependent upon the kinetics of the dominant defect mechanism. We identify a transition between dislocation and twin dominated spall of [001] single crystals at $10^{9} \mathrm{~s}^{-1}$ and show that small nanocrystals $(\mathrm{d}=21 \mathrm{~nm})$ do not indicate a change in mechanism for strain rates greater than $10^{8} \mathrm{~s}^{-1}$. Above the threshold for de-cohesion, $\sim 5 \times 10^{10} \mathrm{~s}^{-1}$, strength again follows a power law dependence up until the vibrational frequency, after which the 
stress saturates at an ultimate value. In each spall process, the temperature induced via plasticity

(and the shock Hugoniot for NEMD simulations) decreases the resulting spall strength. Softening persists up until melting where the spall strength of liquid decreases further in proportion to $\sim 1 / \mathrm{T}$. The classical cleavage strength of tantalum is $39.4 \mathrm{GPa}$ based upon a cohesive energy criterion $[84,85]$. Several potential forms were assessed in order to more accurately estimate the tensile strength. The maximum predicted strength of the Ravelo et al. [33] potential utilized here (Ta1) is $31.6 \mathrm{GPa}$ (Ta2 predicts a theoretical strength of $35 \mathrm{GPa}$ ). These both show good agreement with DFT and general EOS forms, and with the measured ultimate tensile strengths in the current MD simulations of single and nanocrystalline tantalum, $\sim 31$ and $\sim 34 \mathrm{GPa}$, respectively.

\section{Acknowledgments}

ENH and MAM were supported by UC Research Laboratories Grant (09-LR-06-118456-MEYM) and by the U.S. Department of Energy (DOE) NNSA/SSAP (DE-NA0002080). ENH, RR, and TCG received support from the DOE Office of Science, Office of Advanced Scientific Computing Research through the Exascale Co-design Center for Materials in Extreme Environments (ExMatEx). RR acknowledges support from the Air Force Office of Scientific Research under Award FA9550-12-1-0476. Los Alamos National Laboratory, an affirmative action/equal opportunity employer, is operated by Los Alamos National Security, LLC, for the National Nuclear Security Administration of the U.S. Department of Energy under contract DE-AC52-06NA25396. Many useful discussions with Bruce Remington, Chris Wehrenberg, Eduardo Bringa, Diego Tramontina, Tane Remington, and Shiteng Zhao are acknowledged.

\section{References}

[1] T. Antoun, Spall Fracture, Springer New York, 2003.

[2] V.A. Lubarda, M.S. Schneider, D.H. Kalantar, B.A. Remington, M.A. Meyers, Void growth by dislocation emission, Acta Mater. 52 (2004) 1397-1408. doi:10.1016/j.actamat.2003.11.022.

[3] J. Belak, On the nucleation and growth of voids at high strain-rates, J. Comput.-Aided Mater. Des. 5 (1998) 193-206. doi:10.1023/A:1008685029849.

[4] M.A. Meyers, C.T. Aimone, Dynamic fracture (spalling) of metals, Prog. Mater. Sci. 28 (1983) 196. doi:10.1016/0079-6425(83)90003-8.

[5] B. Hopkinson, A Method of Measuring the Pressure Produced in the Detonation of High Explosives or by the Impact of Bullets, Philos. Trans. R. Soc. Lond. Ser. Contain. Pap. Math. Phys. Character. 213 (1914) 437-456. 
[6] L. Davison, A.L. Stevens, M.E. Kipp, Theory of spall damage accumulation in ductile metals, J. Mech. Phys. Solids. 25 (1977) 11-28. doi:10.1016/0022-5096(77)90017-5.

[7] D.E. Grady, The spall strength of condensed matter, J. Mech. Phys. Solids. 36 (1988) 353-384. doi:10.1016/0022-5096(88)90015-4.

[8] V.E. Fortov, V.V. Kostin, S. Eliezer, Spallation of metals under laser irradiation, J. Appl. Phys. 70 (1991) 4524-4531. doi:10.1063/1.349087.

[9] S.V. Razorenov, G.I. Kanel, G.V. Garkushin, O.N. Ignatova, Resistance to dynamic deformation and fracture of tantalum with different grain and defect structures, Phys. Solid State. 54 (2012) 790797. doi:10.1134/S1063783412040233.

[10] J.P. Cuq-Lelandais, M. Boustie, L. Berthe, T. de Rességuier, P. Combis, J.P. Colombier, M. Nivard, A. Claverie, Spallation generated by femtosecond laser driven shocks in thin metallic targets, J. Phys. Appl. Phys. 42 (2009) 65402. doi:10.1088/0022-3727/42/6/065402.

[11] S.I. Ashitkov, P.S. Komarov, E.V. Struleva, M.B. Agranat, G.I. Kanel, K.V. Khishchenko, The behavior of tantalum under ultrashort loads induced by femtosecond laser, J. Phys. Conf. Ser. 653 (2015) 12001. doi:10.1088/1742-6596/653/1/012001.

[12] E. Moshe, S. Eliezer, E. Dekel, A. Ludmirsky, Z. Henis, M. Werdiger, I.B. Goldberg, N. Eliaz, D. Eliezer, An increase of the spall strength in aluminum, copper, and Metglas at strain rates larger than 107 s-1, J. Appl. Phys. 83 (1998) 4004-4011. doi:10.1063/1.367222.

[13] G.I. Kanel, Unusual behaviour of usual materials in shock waves, J. Phys. Conf. Ser. 500 (2014) 12001. doi:10.1088/1742-6596/500/1/012001.

[14] E. Dekel, S. Eliezer, Z. Henis, E. Moshe, A. Ludmirsky, I.B. Goldberg, Spallation model for the high strain rates range, J. Appl. Phys. 84 (1998) 4851-4858. doi:10.1063/1.368727.

[15] S.J. Fensin, E.K. Cerreta, G.T. Gray III, S.M. Valone, Why are some Interfaces in Materials Stronger than others?, Sci. Rep. 4 (2014) 5461. doi:10.1038/srep05461.

[16] K. Mackenchery, R.R. Valisetty, R.R. Namburu, A. Stukowski, A.M. Rajendran, A.M. Dongare, Dislocation evolution and peak spall strengths in single crystal and nanocrystalline $\mathrm{Cu}, \mathrm{J}$. Appl. Phys. 119 (2016) 44301. doi:10.1063/1.4939867.

[17] S. Christy, H. Pak, M. Meyers, Metallurgical applications of shock wave and high-strain-rate phenomena, N. Y. Basel. (1986).

[18] M.A. Meyers, R.W. Armstrong, H.O. Kirchner, Mechanics and materials: fundamentals and linkages, Wiley-VCH, 1999.

[19] A.K. Zurek, M.A. Meyers, Microstructural aspects of dynamic failure, in: High-Press. Shock Compression Solids II, Springer, 1996: pp. 25-70.

[20] L. Tollier, R. Fabbro, Study of the laser-driven spallation process by the VISAR interferometry technique. II. Experiment and simulation of the spallation process, J. Appl. Phys. 83 (1998) 12311237. doi:10.1063/1.366820.

[21] D. Milathianaki, S. Boutet, G.J. Williams, A. Higginbotham, D. Ratner, A.E. Gleason, M. Messerschmidt, M.M. Seibert, D.C. Swift, P. Hering, J. Robinson, W.E. White, J.S. Wark, Femtosecond Visualization of Lattice Dynamics in Shock-Compressed Matter, Science. 342 (2013) 220-223. doi:10.1126/science.1239566.

[22] C.E. Wehrenberg, A.J. Comley, N.R. Barton, F. Coppari, D. Fratanduono, C.M. Huntington, B.R. Maddox, H.-S. Park, C. Plechaty, S.T. Prisbrey, B.A. Remington, R.E. Rudd, Lattice-level observation of the elastic-to-plastic relaxation process with subnanosecond resolution in shockcompressed Ta using time-resolved Itextit\{in situ\} Laue diffraction, Phys. Rev. B. 92 (2015) 104305. doi:10.1103/PhysRevB.92.104305.

[23] P.A. Rigg, R.J. Scharff, R.S. Hixson, Sound speed measurements in tantalum using the front surface impact technique, J. Phys. Conf. Ser. 500 (2014) 32018. doi:10.1088/1742-6596/500/3/032018.

[24] L. Tollier, R. Fabbro, E. Bartnicki, Study of the laser-driven spallation process by the velocity interferometer system for any reflector interferometry technique. I. Laser-shock characterization, J. Appl. Phys. 83 (1998) 1224-1230. doi:10.1063/1.366819. 
[25] D. Chen, Y. Yu, Z. Yin, H. Wang, G. Liu, On the validity of the traditional measurement of spall strength, Int. J. Impact Eng. 31 (2005) 811-824. doi:10.1016/j.ijimpeng.2004.04.006.

[26] G.T. Gray III, N.K. Bourne, V. Livescu, C.P. Trujillo, S. MacDonald, P. Withers, The influence of shock-loading path on the spallation response of Ta, J. Phys. Conf. Ser. 500 (2014) 112031. doi:10.1088/1742-6596/500/11/112031.

[27] O.T. Kingstedt, J. Lambros, Ultra-high Speed Imaging of Laser-Induced Spallation, Exp. Mech. 55 (2015) 587-598. doi:10.1007/s11340-014-9973-0.

[28] C. Czarnota, S. Mercier, A. Molinari, Modelling of nucleation and void growth in dynamic pressure loading, application to spall test on tantalum, Int. J. Fract. 141 (2006) 177-194. doi:10.1007/s10704-006-0070-y.

[29] C. Bronkhorst, G. Gray III, F. Addessio, V. Livescu, N. Bourne, S. MacDonald, P. Withers, Response and representation of ductile damage under varying shock loading conditions in tantalum, J. Appl. Phys. 119 (2016) 85103.

[30] E.M. Bringa, A. Caro, Y. Wang, M. Victoria, J.M. McNaney, B.A. Remington, R.F. Smith, B.R. Torralva, H.V. Swygenhoven, Ultrahigh Strength in Nanocrystalline Materials Under Shock Loading, Science. 309 (2005) 1838-1841. doi:10.1126/science.1116723.

[31] R.E. Rudd, T.C. Germann, B.A. Remington, J.S. Wark, Metal deformation and phase transitions at extremely high strain rates, MRS Bull. 35 (2010) 999-1006. doi:10.1557/mrs2010.705.

[32] B.L. Holian, P.S. Lomdahl, Plasticity Induced by Shock Waves in Nonequilibrium MolecularDynamics Simulations, Science. 280 (1998) 2085-2088. doi:10.1126/science.280.5372.2085.

[33] R. Ravelo, T.C. Germann, O. Guerrero, Q. An, B.L. Holian, Shock-induced plasticity in tantalum single crystals: Interatomic potentials and large-scale molecular-dynamics simulations, Phys. Rev. B. 88 (2013) 134101. doi:10.1103/PhysRevB.88.134101.

[34] D. Tramontina, P. Erhart, T. Germann, J. Hawreliak, A. Higginbotham, N. Park, R. Ravelo, A. Stukowski, M. Suggit, Y. Tang, J. Wark, E. Bringa, Molecular dynamics simulations of shockinduced plasticity in tantalum, High Energy Density Phys. 10 (2014) 9-15. doi:10.1016/j.hedp.2013.10.007.

[35] N.J. Wagner, B.L. Holian, A.F. Voter, Molecular-dynamics simulations of two-dimensional materials at high strain rates, Phys. Rev. A. 45 (1992) 8457-8470. doi:10.1103/PhysRevA.45.8457.

[36] S.-N. Luo, Q. An, T.C. Germann, L.-B. Han, Shock-induced spall in solid and liquid Cu at extreme strain rates, J. Appl. Phys. 106 (2009) 13502. doi:10.1063/1.3158062.

[37] S.-N. Luo, T.C. Germann, D.L. Tonks, Spall damage of copper under supported and decaying shock loading, J. Appl. Phys. 106 (2009) 123518. doi:10.1063/1.3271414.

[38] S.-N. Luo, T.C. Germann, D.L. Tonks, Q. An, Shock wave loading and spallation of copper bicrystals with asymmetric $\Sigma 3\langle 110\rangle$ tilt grain boundaries, J. Appl. Phys. 108 (2010) 93526. doi:10.1063/1.3506707.

[39] C. Wu, L.V. Zhigilei, Nanocrystalline and Polyicosahedral Structure of a Nanospike Generated on Metal Surface Irradiated by a Single Femtosecond Laser Pulse, J. Phys. Chem. C. 120 (2016) 44384447. doi:10.1021/acs.jpcc.6b00013.

[40] S.G. Srinivasan, M.I. Baskes, G.J. Wagner, Atomistic simulations of shock induced microstructural evolution and spallation in single crystal nickel, J. Appl. Phys. 101 (2007) 43504. doi:10.1063/1.2423084.

[41] N. Gunkelmann, E.M. Bringa, H.M. Urbassek, Influence of phase transition on shock-induced spallation in nanocrystalline iron, J. Appl. Phys. 118 (2015) 185902. doi:10.1063/1.4935452.

[42] A. Strachan, T. Çağın, W. Goddard, Critical behavior in spallation failure of metals, Phys. Rev. B. 63 (2001). doi:10.1103/PhysRevB.63.060103.

[43] J.-P. Cuq-Lelandais, M. Boustie, L. Soulard, L. Berthe, A. Sollier, J. Bontaz-Carion, P. Combis, T. de Rességuier, E. Lescoute, Comparison between Experiments and Molecular Dynamic Simulations of Spallation Induced by Ultra-Short Laser Shock on Micrometric Tantalum Targets, AIP Conf. Proc. 1195 (2009) 829-832. doi:10.1063/1.3295270. 
[44] L. Soulard, J. Bontaz-Carion, J.P. Cuq-Lelandais, Experimental and numerical study of the tantalum single crystal spallation, Eur. Phys. J. B. 85 (2012) 1-15. doi:10.1140/epjb/e2012-30269-9.

[45] R.E. Rudd, Void growth in bec metals simulated with molecular dynamics using the Finnis-Sinclair potential, Philos. Mag. 89 (2009) 3133-3161.

[46] E.M. Bringa, S. Traiviratana, M.A. Meyers, Void initiation in fcc metals: Effect of loading orientation and nanocrystalline effects, Acta Mater. 58 (2010) 4458-4477. doi:10.1016/j.actamat.2010.04.043.

[47] C.J. Ruestes, E.M. Bringa, A. Stukowski, J.F. Rodríguez Nieva, Y. Tang, M.A. Meyers, Plastic deformation of a porous bcc metal containing nanometer sized voids, Comput. Mater. Sci. 88 (2014) 92-102. doi:10.1016/j.commatsci.2014.02.047.

[48] Y. Tang, E.M. Bringa, M.A. Meyers, Ductile tensile failure in metals through initiation and growth of nanosized voids, Acta Mater. 60 (2012) 4856-4865. doi:10.1016/j.actamat.2012.05.030.

[49] Y. Tang, E.M. Bringa, B.A. Remington, M.A. Meyers, Growth and collapse of nanovoids in tantalum monocrystals, Acta Mater. 59 (2011) 1354-1372. doi:10.1016/j.actamat.2010.11.001.

[50] Q. An, R. Ravelo, T.C. Germann, W.Z. Han, S.-N.N. Luo, D.L. Tonks, I.I.I. W. A. Goddard, Shock compression and spallation of single crystal tantalum, AIP Conf. Proc. 1426 (2012) 1259-1262. doi:10.1063/1.3686509.

[51] D.R. Curran, L. Seaman, D.A. Shockey, Dynamic failure in solids, Phys. Today. 30 (2008) 46-55. doi:10.1063/1.3037367.

[52] E. Orowan, A type of plastic deformation new in metals, Nature. 149 (1942) 643-644.

[53] R.F. Smith, R.W. Minich, R.E. Rudd, J.H. Eggert, C.A. Bolme, S.L. Brygoo, A.M. Jones, G.W. Collins, Orientation and rate dependence in high strain-rate compression of single-crystal silicon, Phys. Rev. B. 86 (2012) 245204. doi:10.1103/PhysRevB.86.245204.

[54] C.-H. Lu, E.N. Hahn, B.A. Remington, B.R. Maddox, E.M. Bringa, M.A. Meyers, Phase Transformation in Tantalum under Extreme Laser Deformation, Sci. Rep. 5 (2015) 15064. doi:10.1038/srep15064.

[55] E.N. Hahn, T.C. Germann, R.J. Ravelo, J.E. Hammerberg, M.A. Meyers, Non-Equilibrium Molecular Dynamics Simulations of Spall in Single Crystal Tantalum, AIP Conf. Proc. In press (2016).

[56] J.W. Swegle, D.E. Grady, Shock viscosity and the prediction of shock wave rise times, J. Appl. Phys. 58 (1985) 692-701. doi:10.1063/1.336184.

[57] L.E. Murr, M.A. Meyers, C.-S. Niou, Y.J. Chen, S. Pappu, C. Kennedy, Shock-induced deformation twinning in tantalum, Acta Mater. 45 (1997) 157-175. doi:10.1016/S1359-6454(96)00145-0.

[58] K. Kadau, T.C. Germann, P. Lomdahl, Molecular Dynamics Comes of Age: 320 Billion Atom Simulation on Bluegene/L, Int. J. Mod. Phys. C. 17 (2006) 1755-1761. doi:10.1142/S0129183106010182.

[59] P.S. Lomdahl, D.M. Beazley, P. Tamayo, N. Gronbech-Jensen, Multi-Million Particle Molecular Dynamics on The CM-5, Int. J. Mod. Phys. C. 4 (1993) 1075-1084. doi:10.1142/S0129183193000835.

[60] P.S. Lomdahl, P. Tamayo, N. Gronbech-Jensen, D.M. Beazley, 50 GFlops molecular dynamics on the Connection Machine-5, in: Supercomput. 93 Proc., 1993: pp. 520-527. doi:10.1109/SUPERC.1993.1263501.

[61] S. Plimpton, Fast Parallel Algorithms for Short-Range Molecular Dynamics, J. Comput. Phys. 117 (1995) 1-19. doi:10.1006/jcph.1995.1039.

[62] R. Ravelo, Q. An, T.C. Germann, B.L. Holian, Large-scale molecular dynamics simulations of shock induced plasticity in tantalum single crystals, AIP Conf. Proc. 1426 (2012) 1263-1266. doi:10.1063/1.3686510.

[63] S.J. Plimpton, A.P. Thompson, Computational aspects of many-body potentials, MRS Bull. 37 (2012) 513-521. doi:10.1557/mrs.2012.96. 
[64] A. Stukowski, Visualization and analysis of atomistic simulation data with OVITO-the Open Visualization Tool, Model. Simul. Mater. Sci. Eng. 18 (2010) 15012. doi:10.1088/09650393/18/1/015012.

[65] A. Stukowski, K. Albe, Extracting dislocations and non-dislocation crystal defects from atomistic simulation data, Model. Simul. Mater. Sci. Eng. 18 (2010) 85001. doi:10.1088/09650393/18/8/085001.

[66] A. Stukowski, Computational Analysis Methods in Atomistic Modeling of Crystals, JOM. 66 (2013) 399-407. doi:10.1007/s11837-013-0827-5.

[67] A.P. Thompson, S.J. Plimpton, W. Mattson, General formulation of pressure and stress tensor for arbitrary many-body interaction potentials under periodic boundary conditions, J. Chem. Phys. 131 (2009) 154107. doi:10.1063/1.3245303.

[68] B.L. Boyce, B.G. Clark, P. Lu, J.D. Carroll, C.R. Weinberger, The Morphology of Tensile Failure in Tantalum, Metall. Mater. Trans. A. 44 (2013) 4567-4580. doi:10.1007/s11661-013-1814-8.

[69] E.N. Hahn, S.J. Fensin, T.C. Germann, M.A. Meyers, Symmetric tilt boundaries in body-centered cubic tantalum, Scr. Mater. 116 (2016) 108-111. doi:10.1016/j.scriptamat.2016.01.038.

[70] Z. Shi, C.V. Singh, Competing twinning mechanisms in body-centered cubic metallic nanowires, Scr. Mater. 113 (2016) 214-217. doi:10.1016/j.scriptamat.2015.11.006.

[71] H.-J. Chang, J. Segurado, O.R. de la Fuente, B.M. Pabón, J. LLorca, Molecular dynamics modeling and simulation of void growth in two dimensions, Model. Simul. Mater. Sci. Eng. 21 (2013) 75010. doi:10.1088/0965-0393/21/7/075010.

[72] D.R. Tramontina, E.N. Hahn, M.A. Meyers, E.M. Bringa, Simulation of Tantalum Nanocrystals Under Shock Wave Loading: Dislocations and Twinning, AIP Conf. Proc. In press (2016).

[73] T.P. Remington, C.J. Ruestes, E.M. Bringa, B.A. Remington, C.H. Lu, B. Kad, M.A. Meyers, Plastic deformation in nanoindentation of tantalum: A new mechanism for prismatic loop formation, Acta Mater. 78 (2014) 378-393. doi:10.1016/j.actamat.2014.06.058.

[74] Y. Tang, E.M. Bringa, M.A. Meyers, Inverse Hall-Petch relationship in nanocrystalline tantalum, Mater. Sci. Eng. A. 580 (2013) 414-426. doi:10.1016/j.msea.2013.05.024.

[75] A. Stukowski, Structure identification methods for atomistic simulations of crystalline materials, Model. Simul. Mater. Sci. Eng. 20 (2012) 45021. doi:10.1088/0965-0393/20/4/045021.

[76] R. Ravelo, B.L. Holian, T.C. Germann, High Strain Rates Effects in Quasi-Isentropic Compression of Solids, AIP Conf. Proc. 1195 (2009) 825-828.

[77] A.E. Mayer, P.N. Mayer, Continuum model of tensile fracture of metal melts and its application to a problem of high-current electron irradiation of metals, J. Appl. Phys. 118 (2015) 35903. doi:10.1063/1.4926861.

[78] Y.Y. Boguslavskii, Equation for the Melting Curve of Solids under High Pressure, Phys. Status Solidi B. 109 (1982) 145-151. doi:10.1002/pssb.2221090115.

[79] D.B. Holtkamp, D.A. Clark, E.N. Ferm, R.A. Gallegos, D. Hammon, W.F. Hemsing, G.E. Hogan, V.H. Holmes, N.S.P. King, R. Liljestrand, R.P. Lopez, F.E. Merrill, C.L. Morris, K.B. Morley, M.M. Murray, P.D. Pazuchanics, K.P. Prestridge, J.P. Quintana, A. Saunders, T. Schafer, M.A. Shinas, H.L. Stacy, A Survey of High Explosive-Induced Damage and Spall in Selected Metals Using Proton Radiography, in: AIP Conf. Proc., AIP Publishing, 2004: pp. 477-482. doi:10.1063/1.1780281.

[80] C. Wu, L.V. Zhigilei, Microscopic mechanisms of laser spallation and ablation of metal targets from large-scale molecular dynamics simulations, Appl. Phys. A. 114 (2013) 11-32. doi:10.1007/s00339013-8086-4.

[81] J.H. Rose, J.R. Smith, F. Guinea, J. Ferrante, Universal features of the equation of state of metals, Phys. Rev. B. 29 (1984) 2963-2969. doi:10.1103/PhysRevB.29.2963.

[82] D. Tonks, C.A. Bronkhorst, J. Bingert, M.L. Elert, W.T. Buttler, J.P. Borg, J.L. Jordan, T.J. Vogler, A comparison of calculated damage from square waves and triangular waves, AIP Conf. Proc. 1426 (2012) 1045. 
[83] D. Koller, R. Hixson, G. Gray III, P. Rigg, L. Addessio, E. Cerreta, J. Maestas, C. Yablinsky, Influence of shock-wave profile shape on dynamically induced damage in high-purity copper, $\mathrm{J}$. Appl. Phys. 98 (2005) 103518.

[84] D.E. Grady, The spall strength of condensed matter, J. Mech. Phys. Solids. 36 (1988) 353-384. doi:10.1016/0022-5096(88)90015-4.

[85] H. Jarmakani, B. Maddox, C.T. Wei, D. Kalantar, M.A. Meyers, Laser shock-induced spalling and fragmentation in vanadium, Acta Mater. 58 (2010) 4604-4628. doi:10.1016/j.actamat.2010.04.027.

[86] J.N. Johnson, R.S. Hixson, D.L. Tonks, A.K. Zurek, Rate-dependent spallation properties of tantalum, AIP Conf. Proc. 370 (1996) 523-526. doi:10.1063/1.50651.

[87] A.K. Zurek, W.R. Thissell, J.N. Johnson, D.L. Tonks, R. Hixson, Micromechanics of spall and damage in tantalum, J. Mater. Process. Technol. 60 (1996) 261-267. doi:10.1016/09240136(96)02340-0.

[88] J.M. Rivas, A.K. Zurek, W.R. Thissell, D.L. Tonks, R.S. Hixson, Quantitative description of damage evolution in ductile fracture of tantalum, Metall. Mater. Trans. A. 31 (2000) 845-851. doi:10.1007/s11661-000-1004-3.

[89] G. Roy, Vers une modélisation approfondie de l'endommagement ductile dynamique: investigation expérimentale d'une nuance de tantale et développements théoriques, Ph.D. Thesis, Poitiers, 2003.

[90] J.C.F. Millett, G. Whiteman, N.T. Park, S. Case, N.K. Bourne, The role of cold work on the shock response of tantalum, J. Appl. Phys. 113 (2013) 233502. doi:10.1063/1.4810896.

[91] S.A. Abrosimov, A.P. Bazhulin, V.V. Voronov, A.A. Geras'kin, I.K. Krasyuk, P.P. Pashinin, A.Y. Semenov, I.A. Stuchebryukhov, K.V. Khishchenko, V.E. Fortov, Specific features of the behaviour of targets under negative pressures created by a picosecond laser pulse, Quantum Electron. 43 (2013) 246-251. doi:10.1070/QE2013v043n03ABEH015106.

[92] B. Glam, M. Werdiger, Y. Horovitz, E. Moshe, S.L. Pistinner, Dynamic strength of tantalum under impact, J. Phys. Conf. Ser. 500 (2014) 112029. doi:10.1088/1742-6596/500/11/112029.

[93] T.P. Remington, Extreme Response in Tension and Compression of Tantalum, Ph.D. Thesis, University of California San Diego, 2015. http://www.escholarship.org/uc/item/42d8330s.

[94] S. Zhao, B. Kad, E.N. Hahn, B.A. Remington, C.E. Wehrenberg, C.M. Huntington, H.-S. Park, E.M. Bringa, K.L. More, M.A. Meyers, Pressure and shear-induced amorphization of silicon, Extreme Mech. Lett. 5 (2015) 74-80. doi:10.1016/j.eml.2015.10.001.

[95] Z.-L. Liu, L.-C. Cai, X.-R. Chen, Q. Wu, F.-Q. Jing, Ab initio refinement of the thermal equation of state for bcc tantalum: the effect of bonding on anharmonicity, J. Phys. Condens. Matter. 21 (2009) 95408. doi:10.1088/0953-8984/21/9/095408. 
1 Table I. Simulations of single crystalline tantalum. Measurements at the peak tensile stress in the 2 shock direction $\left(\sigma_{\mathrm{zz}}\right)$ and the corresponding hydrostatic pressure $(\mathrm{P})$, deviatoric shear $\operatorname{stress}(\tau)$, 3 temperature $(\mathrm{T})$, and strain $(\varepsilon)$.

\begin{tabular}{rccccccc}
4 & Method & $\dot{\varepsilon}\left(\mathrm{s}^{-1}\right)$ & $\sigma_{\mathrm{zz}}(\mathrm{GPa})$ & $\mathrm{P}(\mathrm{GPa})$ & $\tau(\mathrm{GPa})$ & $\mathrm{T}(\mathrm{K})$ & $\varepsilon$ \\
\cline { 2 - 7 } 5 & QI & $10^{7}$ & 12.1 & 11.7 & 0.29 & 604 & 0.079 \\
6 & QI & $10^{8}$ & 12.8 & 12.2 & 0.51 & 647 & 0.087 \\
7 & QI & $10^{9}$ & 14.0 & 13.0 & 0.72 & 650 & 0.103 \\
8 & QI & $3 \times 10^{9}$ & 15.0 & 13.8 & 0.87 & 642 & 0.117 \\
9 & QI & $10^{10}$ & 15.9 & 14.8 & 0.84 & 644 & 0.139 \\
10 & Piston & $1.65 \times 10^{10}$ & 19.1 & 18.4 & 0.53 & 649 & 0.176 \\
11 & Flyer & $1.75 \times 10^{10}$ & 18.4 & 17.7 & 0.57 & 910 & 0.174 \\
12 & QI & $2 \times 10^{10}$ & 16.9 & 15.8 & 0.76 & 667 & 0.160 \\
13 & QI & $3 \times 10^{10}$ & 17.5 & 16.6 & 0.70 & 701 & 0.179 \\
14 & QI & $6 \times 10^{10}$ & 19.3 & 18.2 & 0.77 & 788 & 0.224 \\
15 & QI & $10^{11}$ & 20.8 & 19.5 & 1.03 & 898 & 0.270 \\
16 & QI & $2 \times 10^{11}$ & 22.9 & 20.7 & 1.67 & 1111 & 0.340 \\
& QI & $3 \times 10^{11}$ & 24.3 & 21.4 & 2.21 & 1174 & 0.380 \\
& QI & $10^{12}$ & 28.2 & 22.8 & 4.04 & 1307 & 0.485 \\
& QI & $10^{13}$ & 31.1 & 22.9 & 6.17 & 619 & 0.565 \\
\hline
\end{tabular}


1 Table II. Simulations of nanocrystalline tantalum, $d=21 \mathrm{~nm}$. Measurements at the peak tensile 2 stress in the shock direction $\left(\sigma_{\mathrm{zz}}\right)$ and the corresponding hydrostatic pressure $(\mathrm{P})$, deviatoric shear $3 \operatorname{stress}(\tau)$, temperature $(\mathrm{T})$, and strain $(\varepsilon)$.

4

5

6

7

8

9

10

\begin{tabular}{ccccccc}
\hline Method & $\dot{\varepsilon}\left(\mathrm{s}^{-1}\right)$ & $\sigma_{\mathrm{zz}}(\mathrm{GPa})$ & $\mathrm{P}(\mathrm{GPa})$ & $\tau(\mathrm{GPa})$ & $\mathrm{T}(\mathrm{K})$ & $\varepsilon$ \\
\hline QI & $10^{8}$ & 8.5 & 6.3 & 1.63 & 500 & 0.057 \\
QI & $10^{9}$ & 10.2 & 7.8 & 1.80 & 523 & 0.070 \\
Piston & $7.8 \times 10^{9}$ & 17.6 & 15.3 & 1.73 & 800 & 0.137 \\
QI & $10^{10}$ & 14.4 & 12.0 & 1.81 & 620 & 0.123 \\
QI & $10^{11}$ & 19.8 & 11.6 & 6.17 & 711 & 0.144 \\
QI & $10^{12}$ & 31.1 & 16.6 & 10.92 & 484 & 0.224 \\
QI & $10^{13}$ & 33.7 & 18.1 & 11.73 & 361 & 0.262 \\
\hline
\end{tabular}

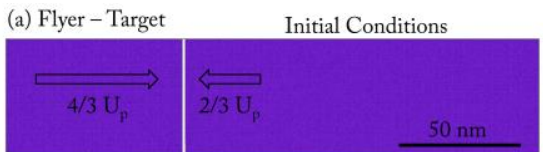

(b) Piston

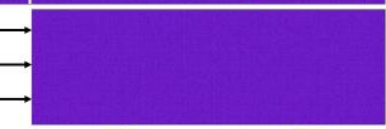

(c) Quasi-Isentropic

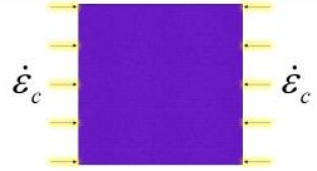

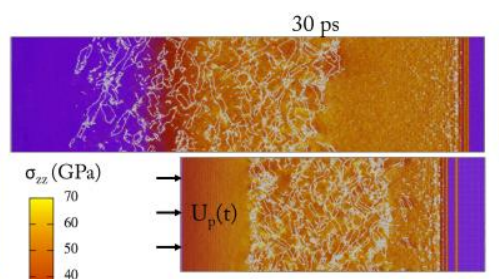

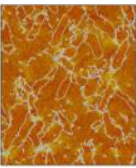

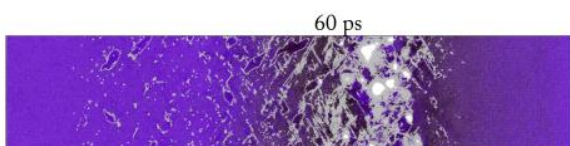
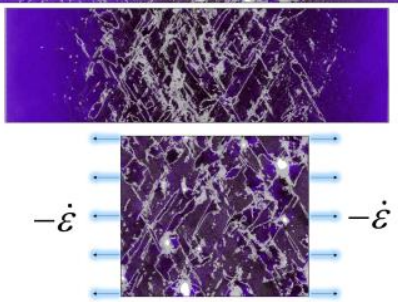

11

12

13

14

15

16

17

Figure 1. Methods used to create high strain-rate compression and release: (a) flyer-target geometry; (b), piston directed compression and release that varies a function of time; and (c) quasi-isentropic compression followed by quasi-isentropic tension. Methods (a) and (b) create shock waves in the system whereas (c) mimics the strain-rate history of a Lagrangian volume in the region of potential spall. 

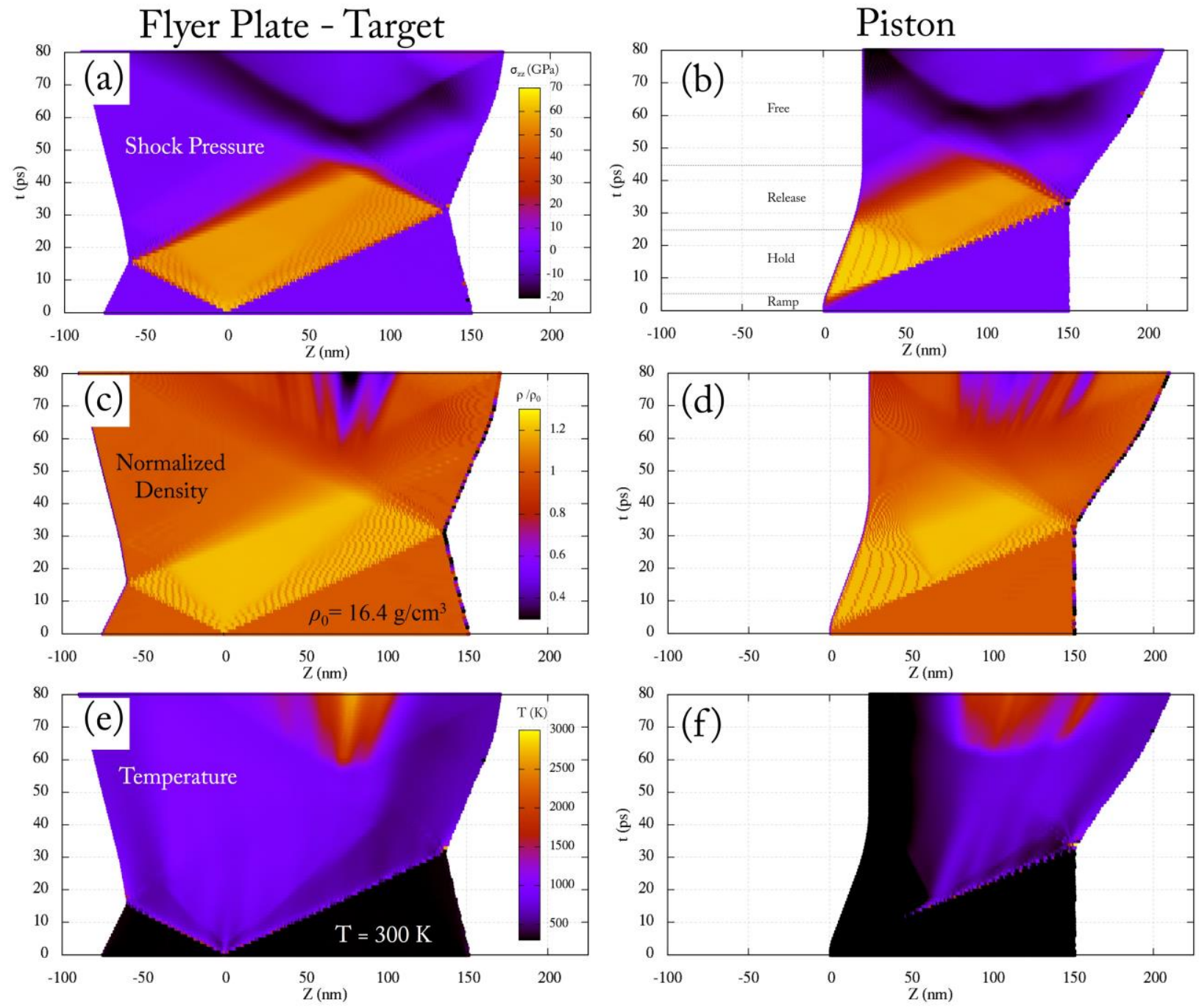

Figure 2. Position-time (x-t) diagrams for the flyer plate and piston compression and release methods. (ab) Longitudinal stress, $\sigma_{\mathrm{zz}}$; (c-d) normalized density. $\rho$; (e-f) temperature, $T$. In panel (b) we indicate the timing of ramp, hold, and release for the piston-driven simulation as they relate to the pressure profile. 


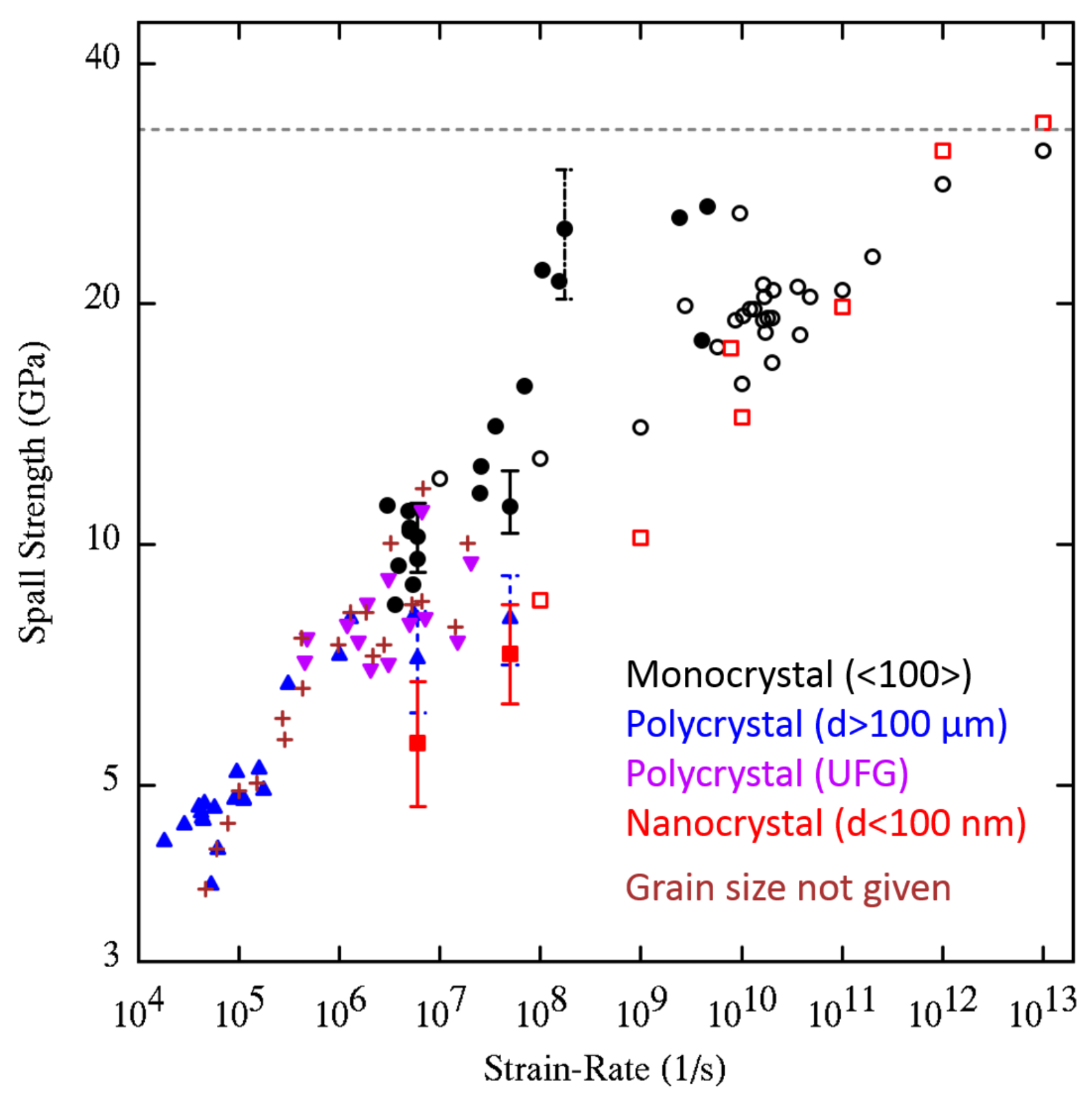

Figure 3. Compilation of reported spall strength as a function of strain rate. Different colors refer to different grain sizes: single crystal (black), polycrystal (blue), nanocrystal (red), ultra-fined grained (purple) and unknown (brown). Data are from multiple sources, including both experimental and simulation work[86$89,10,44,9,50,90,91,26,92,93,11,94]$; experiments are closed symbols and simulations are portrayed as open symbols (with strain rates greater than the phonon frequency marked with crosses). A full set of references is provided in the supplemental material. The ultimate tensile strength is indicated by a horizontal dashed line at $~ 33 \mathrm{GPa}$. 


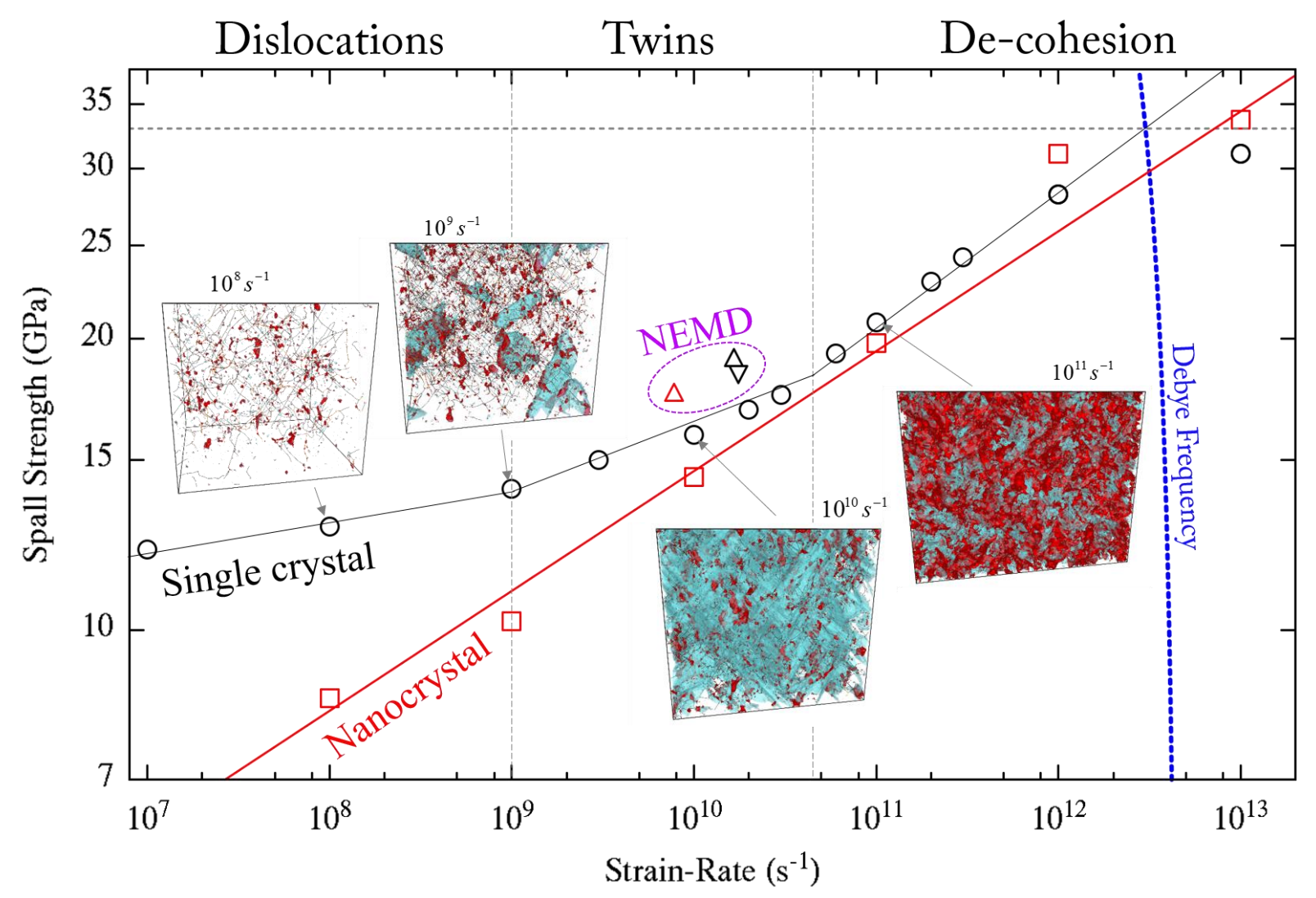

2 Figure 4. Measured spall strength vs strain rate (from Tables I and II). Also plotted is the Debye/phonon 3 frequency on the right hand side of the plot as a dashed blue line; it is expected that the frequency decreases 4 with expansion just as it increases under compression. Phonon frequency as a function of negative pressure was extrapolated from Liu et al.[95]. Snapshots of quasi-isentropic simulations of [001] single crystals at different strain rates - shown at maximum tensile stress. Dislocations are shown as dark lines, twin boundaries as transparent turquoise surfaces, and voids as transparent red surfaces. Regions of dislocation, twin, and de-cohesion dominated spall processes are separated by vertical dashed lines. The strains at which each frame is displayed are provided in Table I and II. NEMD simulations (Piston and Flyer-Target methods) lie off the main lines primarily due to differences in stress state, having lower contributions of 11 shear stresses that aid in nucleating defects. 


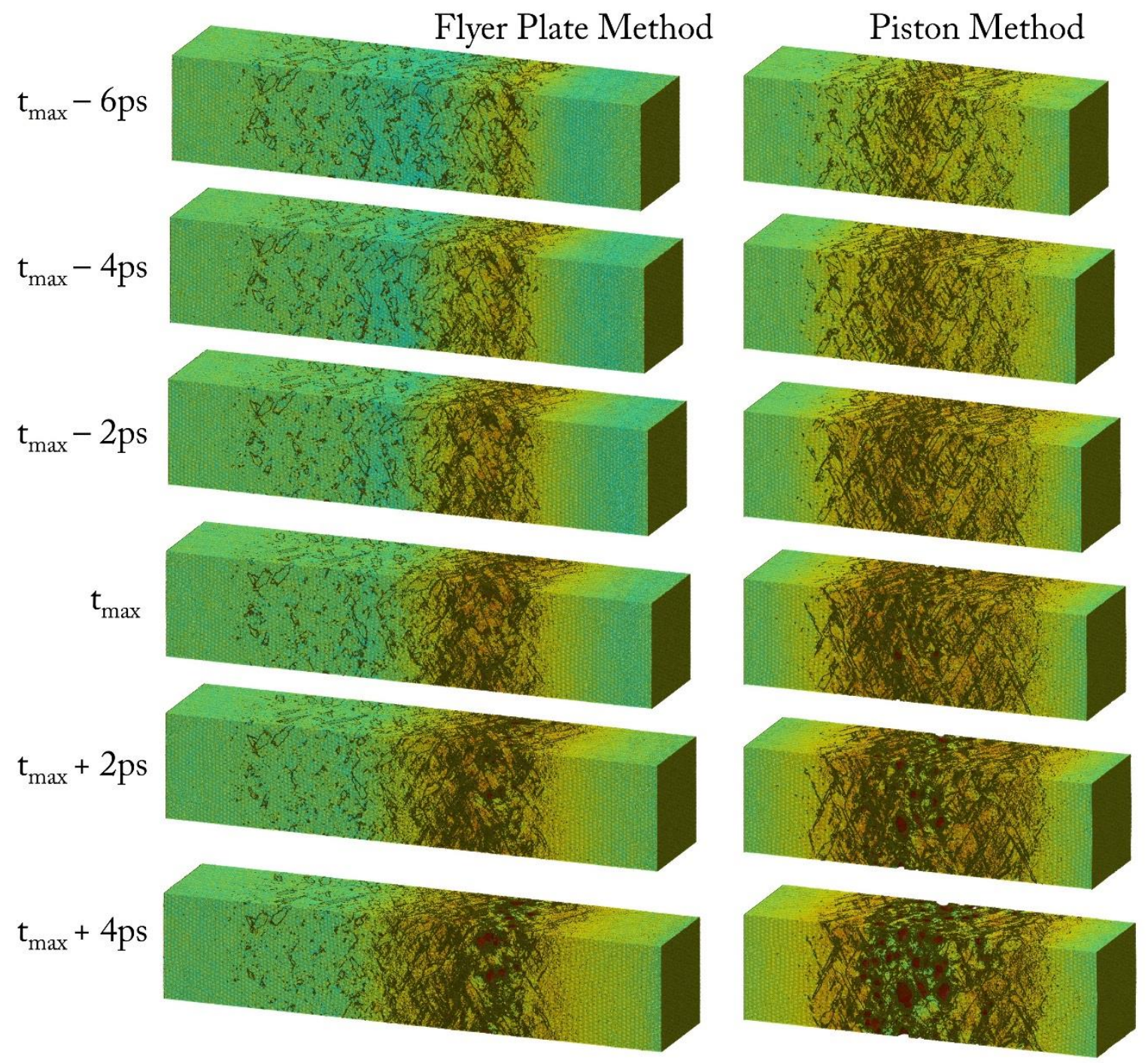

2 Figure 5. Snapshots of the spallation process for flyer plate and piston methods shocked along [001] single 3 crystal Ta. Snapshots are given at the time of maximum tensile stress $\left(\mathrm{t}_{\max }\right)$; and 2, 4, and 6 ps before and 2 4 and $4 \mathrm{ps}$ after this time. For the flyer plate system $\mathrm{t}_{\max }=56 \mathrm{ps}$, and for the piston-driven system $\mathrm{t}_{\max }=61 \mathrm{ps}$. 5 Stress, temperature, and volume profiles are provided in the supplemental material. 

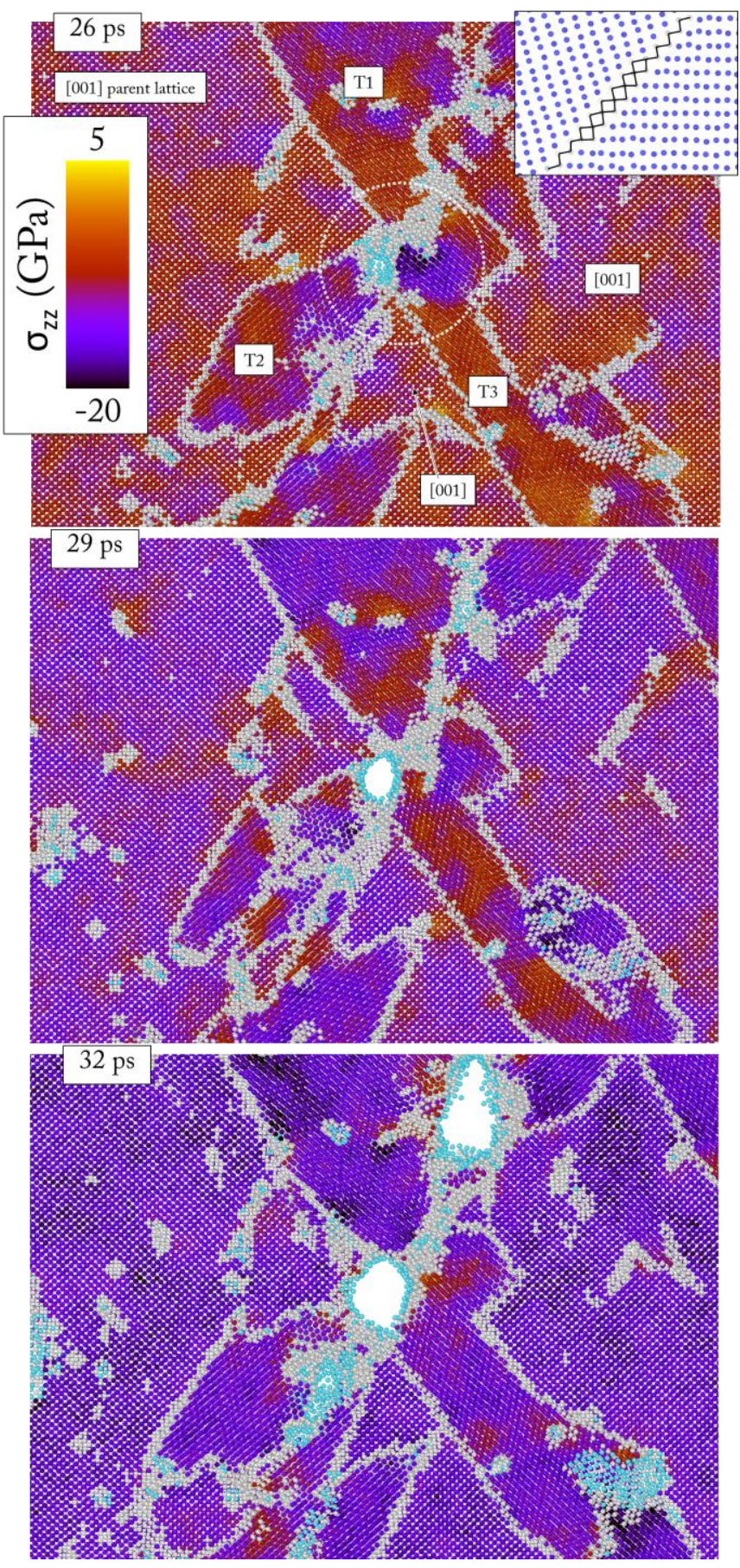

2 Figure 6. (a) Snapshot of twin structures at 26, 29, and 32 ps during QI expansion of a Ta [001] single 3 crystal at a strain rate of $10^{10} \mathrm{~s}^{-1}$. Defective atoms are colored off white and void surfaces are colored light 4 blue. All other atoms are colored according to their local longitudinal stress. The inset in the $26 \mathrm{ps}$ frame 5 shows the atomic configuration of a selected tensile deformation twin with both a symmetric and non6 symmetric $\Sigma 3$ twin boundary, previously shown to be important in the twinning process of bcc metals 7 [70,71]. The first figure also highlights the [001] parent lattice and the intersection of 3 twins which 8 results in a local stress buildup due to compatibility between the twinned and parent orientations. The 9 process illustrated here is directly comparable to the non-equilibrium simulations shown in Fig. 5. 

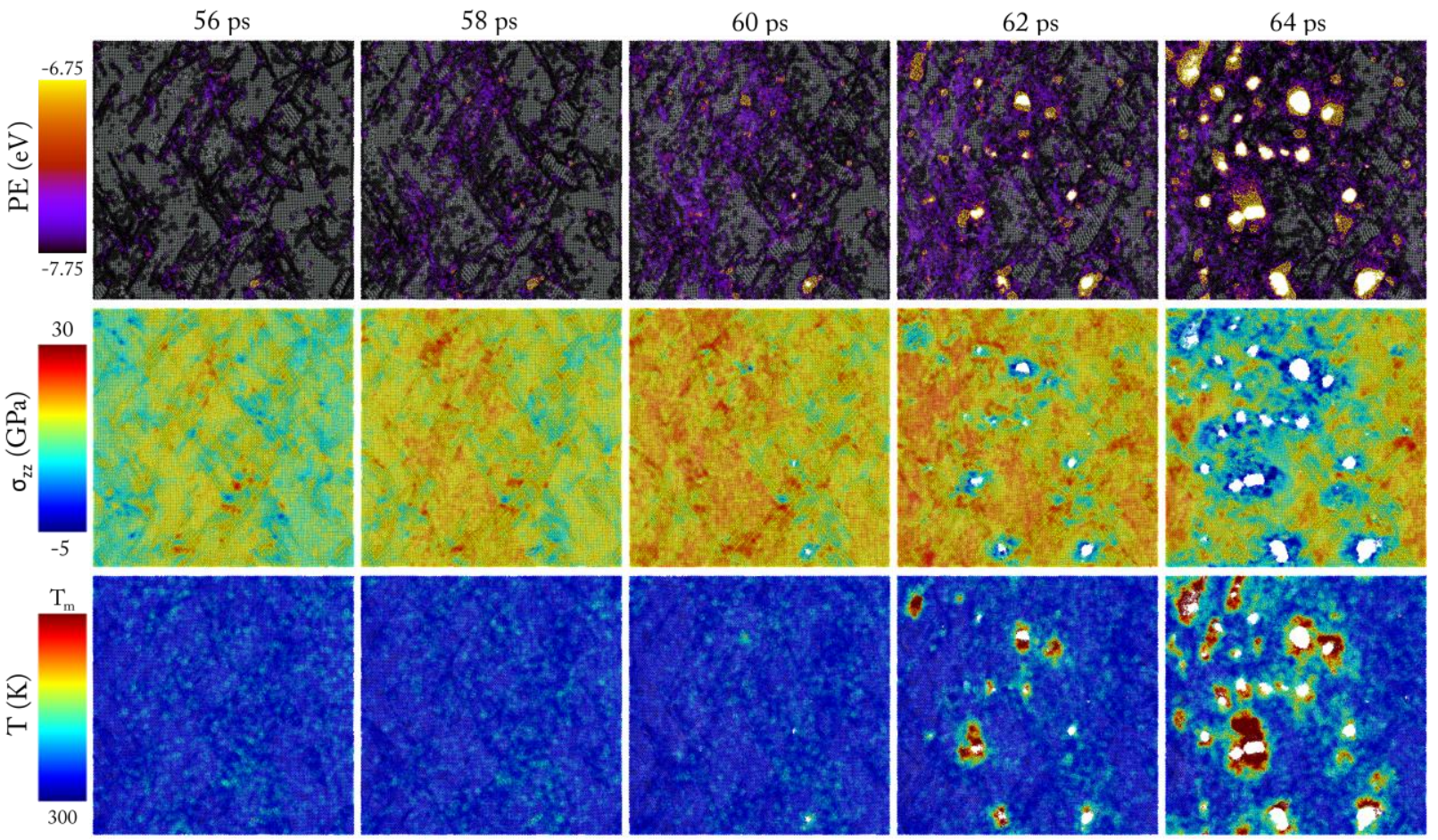

Figure 7. Evolution of voids from the early stages of nucleation, growth, and rapid coalescence. This pistondriven simulation contains a broad tensile pulse that passes through the system traveling to the left as the bulk motion continues to carry the system to the right. Shown in three different visualization schemes is a $50 \mathrm{~nm}$ x $50 \mathrm{~nm}$ transverse cross section $1.5 \mathrm{~nm}$ in thickness centered at $110 \mathrm{~nm}$ along the shock direction. The shock direction is left to right. The top row colors atoms by their local potential energy and allows for precise visualization of atoms belonging to void surfaces (yellow color). The early stages of the void can be seen in the 56 ps timeframe and a surface meshing algorithm[67] is able to detect a void volume at 58 ps using a $0.5 \mathrm{~nm}$ probe sphere radius. The middle row depicts atoms according to their local longitudinal stress value; tensile stress is represented by a positive value (red) and compressive stresses by negative values (blue). The stress value is the average value of the individual atom and neighboring atoms within a $0.66 \mathrm{~nm}$ radius, or a $1.2 \mathrm{~nm}^{3}$ volume. The bottom row presents atoms according to a temperature evaluated for a local neighborhood, again evaluated spherically in a $1.2 \mathrm{~nm}^{3}$ volume. The maximum stress occurs at $61 \mathrm{ps}, 5 \mathrm{ps}$ after the first void nucleated. 


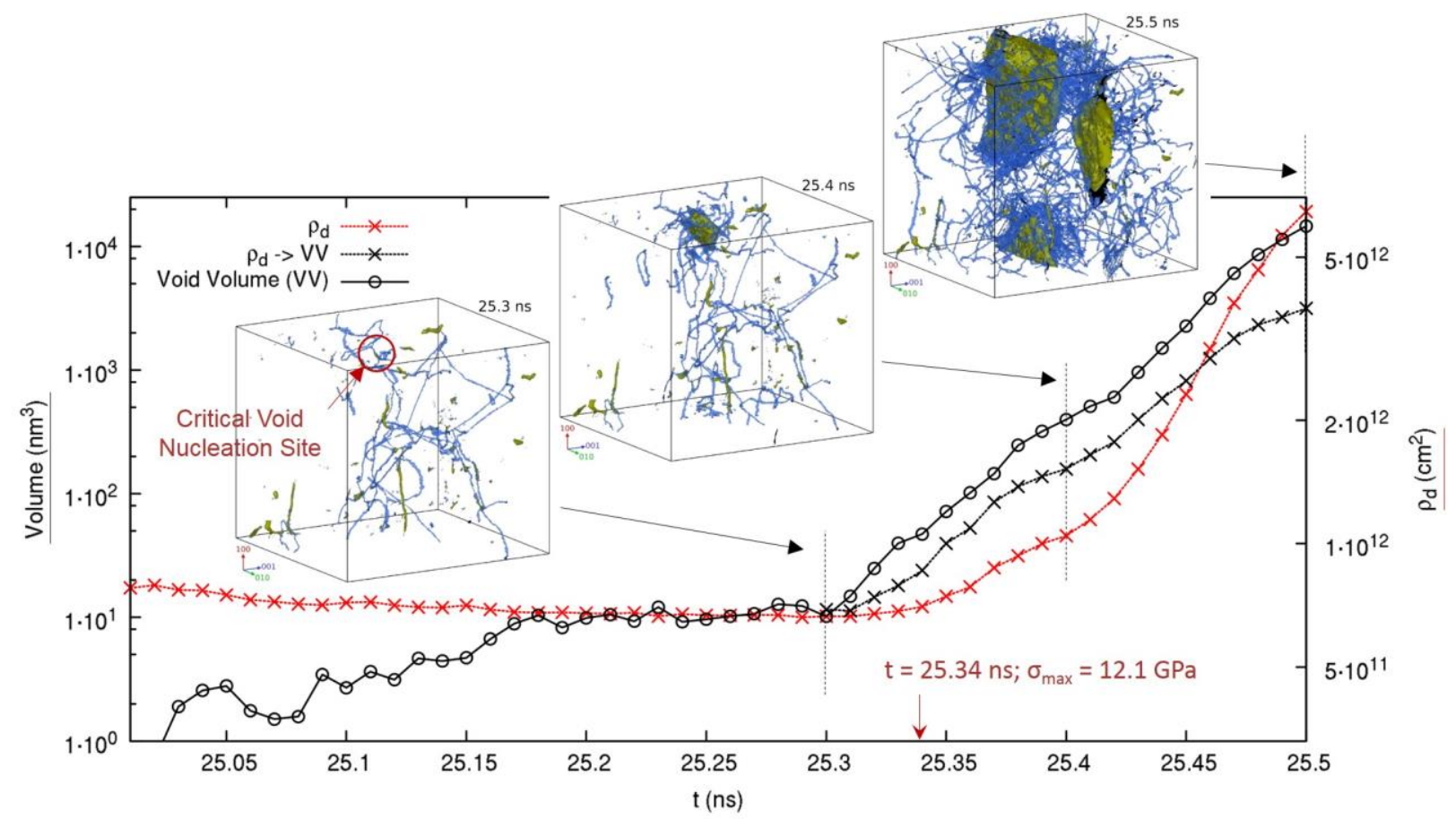

Figure 8. Evolution of porosity and dislocation density during the critical void nucleation and growth event for a strain rate of $10^{7} \mathrm{~s}^{-1}$. Insets show snapshots in time. The critical void nucleation site is along a dislocation running perpendicular to the loading direction. Analysis is halted at $25.5 \mathrm{~ns}$ due to significant interaction of the growing void field across periodic boundaries. Porosity and dislocation line lengths are evaluated using DXA.

(a)

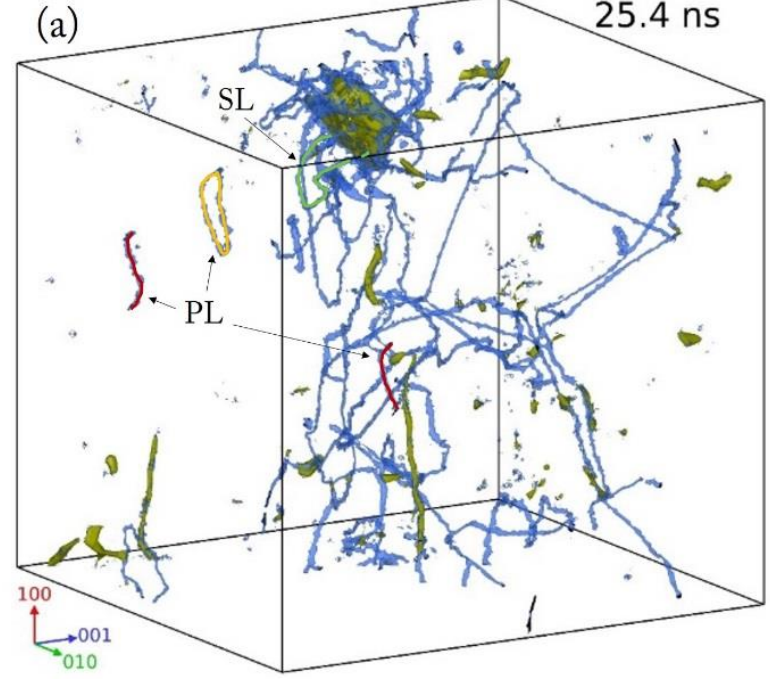

(b)
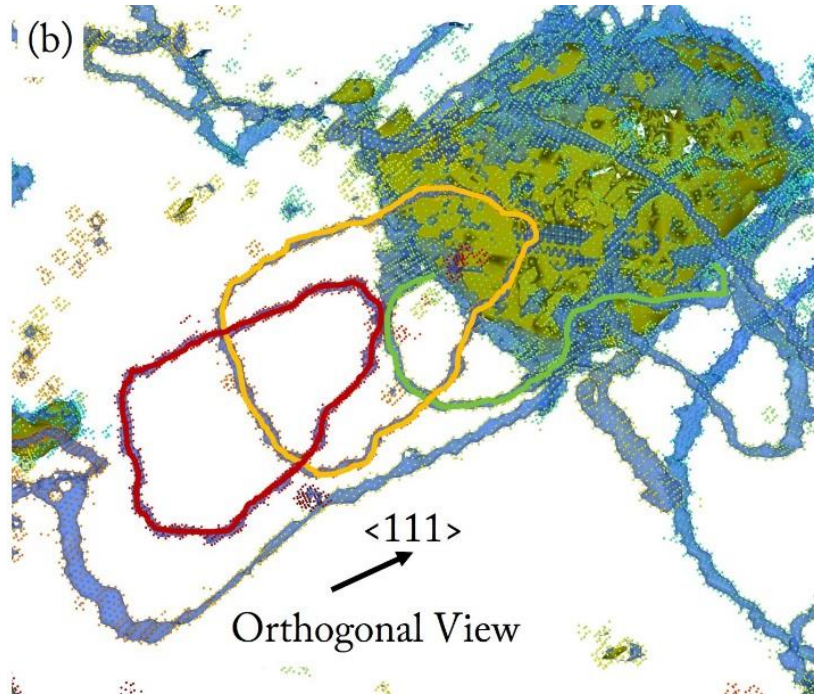

Figure 9. (a) Snapshot of dislocation structures at $25.4 \mathrm{~ns}$ during QI expansion at a strain rate of $10^{7} \mathrm{~s}^{-1}$; larger version of inset within Fig. 8. (b) Orthogonal projection highlighting shear loops (SL) and prismatic loops (PL) and traveling along the $<111>$ direction. This nucleation event has been described previously by Remington et al.[74]. 


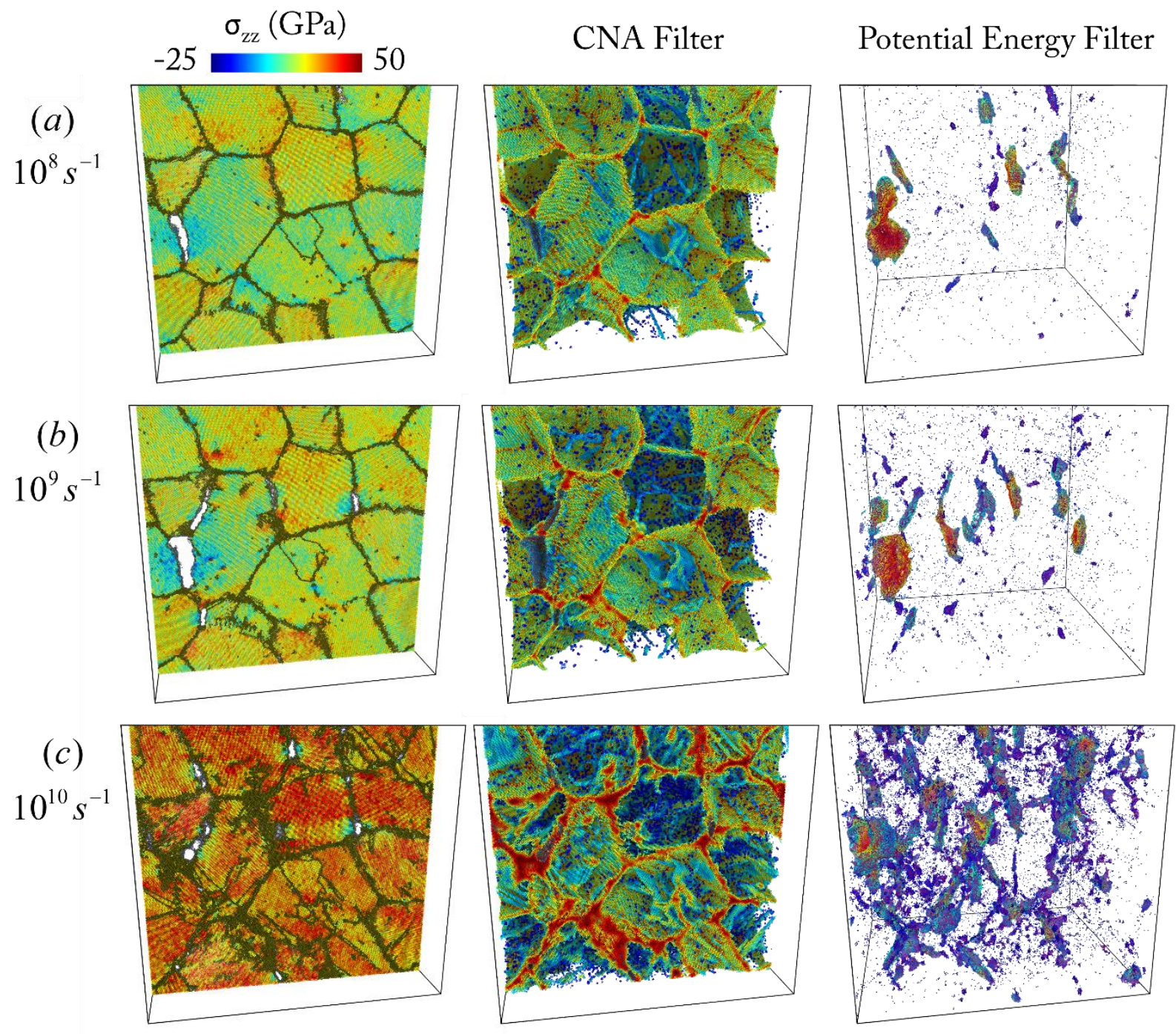

2 Figure 10. Snapshots of quasi-isentropic simulations of nanocrystalline tantalum $(\mathrm{d}=21 \mathrm{~nm})$ at different 3 strain rates, each shown at the time of maximum tension. Strain rates are (a) $10^{8} \mathrm{~s}^{-1}$, (b) $10^{9} \mathrm{~s}^{-1}$, and (c) $10^{10}$ $4 \mathrm{~s}^{-1}$. The strains at which each frame is displayed are provided in Table II. In the first column, atoms are 5 colored according to the local longitudinal stress averaged over $500 \AA$ volumes, positive stresses are tensile 6 and negative stresses are compressive. The second column depicts atoms filtered by common neighbor analysis (CNA) using a $3.9 \AA$ cutoff and colored by "defect coordination," effectively allowing visualization of grain boundaries, dislocations, and twins. The third column shows atoms filtered by a potential energy cutoff of $-7.2 \mathrm{eV}$ which enables the visualization of void surfaces. The third column also utilizes a defect coordination coloring scheme where increasing void volume corresponds to blue, turquoise, yellow, orange and green. This method also allows a qualitative analysis of non-spherical voids; a completely spherical void would exhibit the same color along the entirety of its surface. 


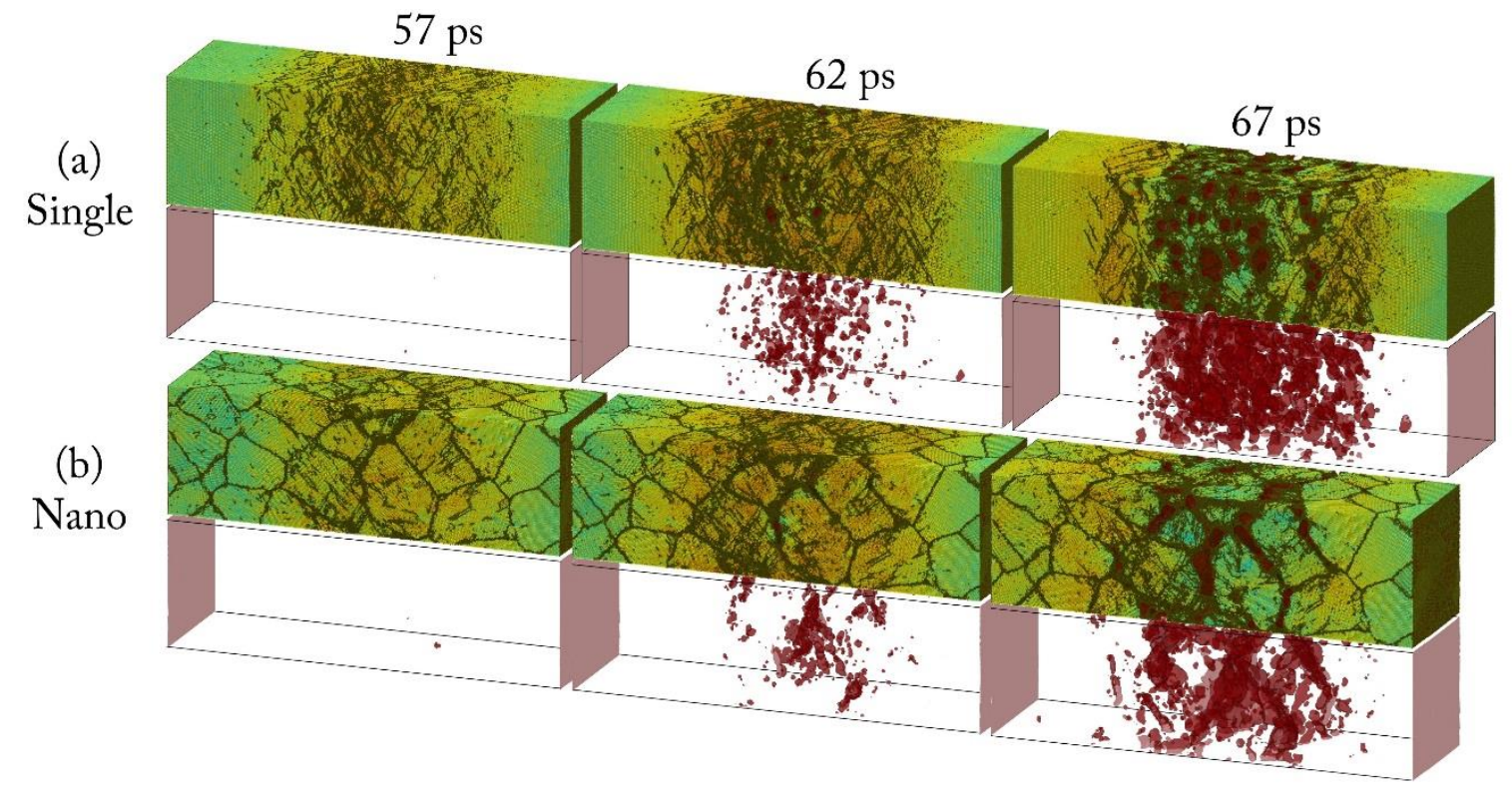

Figure 11. Comparison between single and nanocrystalline spall simulations using the piston methodology. Voids nucleate along the grain boundaries in the nanocrystal, typically at grain boundaries that are perpendicular to the loading direction. The particle velocity is $750 \mathrm{~m} / \mathrm{s}$ corresponding to shock pressures of $56.5 \mathrm{GPa}$ for the [001] shock and $52 \mathrm{GPa}$ for the nanocrystalline shock. The release strain rate is $1.65 \times 10^{10} \mathrm{~s}^{-1}$ for the [001] shock and $7.8 \times 10^{9} \mathrm{~s}^{-1}$ for the nanocrystalline shock. The lower strain rate is due to increased dispersion of the unsupported shock wave in the nanocrystalline sample. 


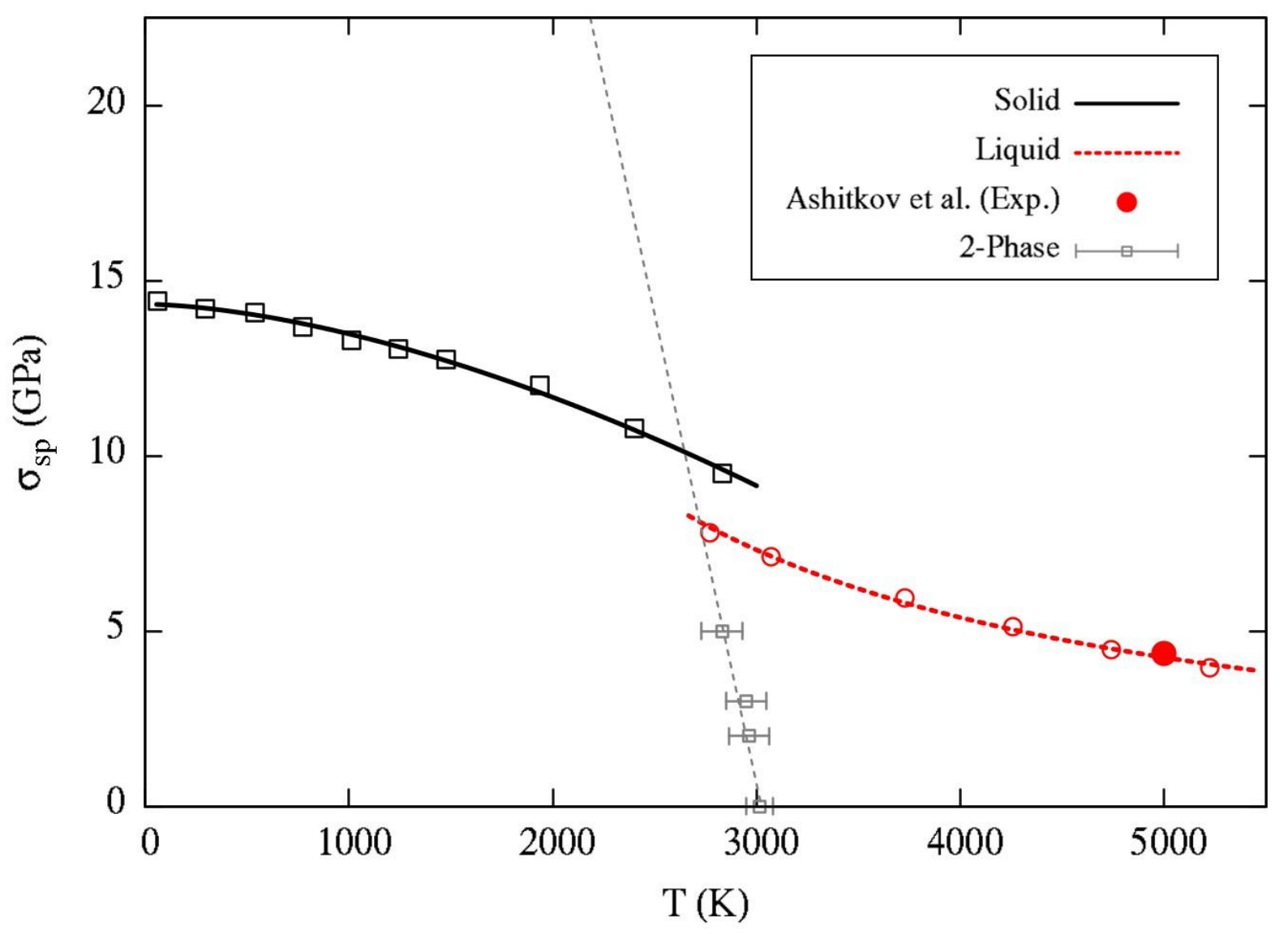

2 Figure 12. Spall strength as a function of temperature at a strain rate of $10^{9} \mathrm{~s}^{-1}$. The melting temperature as 3 a function of pressure was determined from 2 phase simulations (full details can be found in the 4 supplemental material). Ashitkov et al.[11] provides an experimental measurement (solid red circle) for the 5 spall strength (cavitation strength) of liquid tantalum as melted under laser irradiation at an estimated strain 6 rate of $1.5 \times 10^{9} \mathrm{~s}^{-1}$ and a temperature of $\sim 5000 \mathrm{~K}$. 

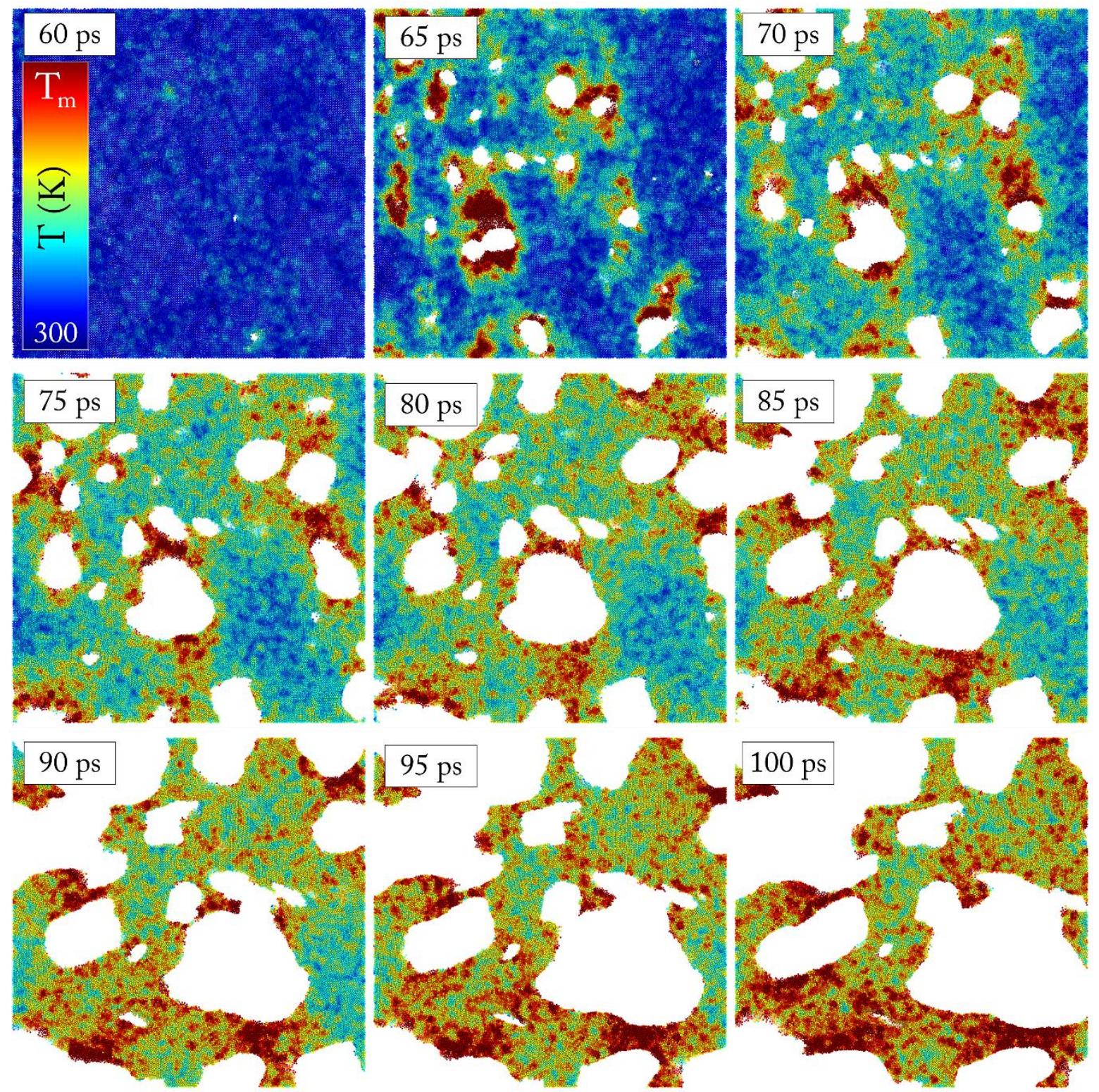

Figure 13. Spall temperature evolution of piston-driven [001] single crystal simulation from 60 to $100 \mathrm{ps}$. $50 \mathrm{~nm}$ x $50 \mathrm{~nm}$ transverse section $1.5 \mathrm{~nm}$ in thickness centered at $110 \mathrm{~nm}$ along the shock direction. The shock direction is right to left and the resulting tensile wave travels from left to right. After initial void nucleation and growth, coalescence is largely affected by regions of high temperature, some of which approach and exceed the melting temperature of tantalum. $\mathrm{T}_{\mathrm{m}}$ is defined as the equilibrium melting temperature of $3033 \mathrm{~K}$, which can be reduced in tension (Fig. 11). 


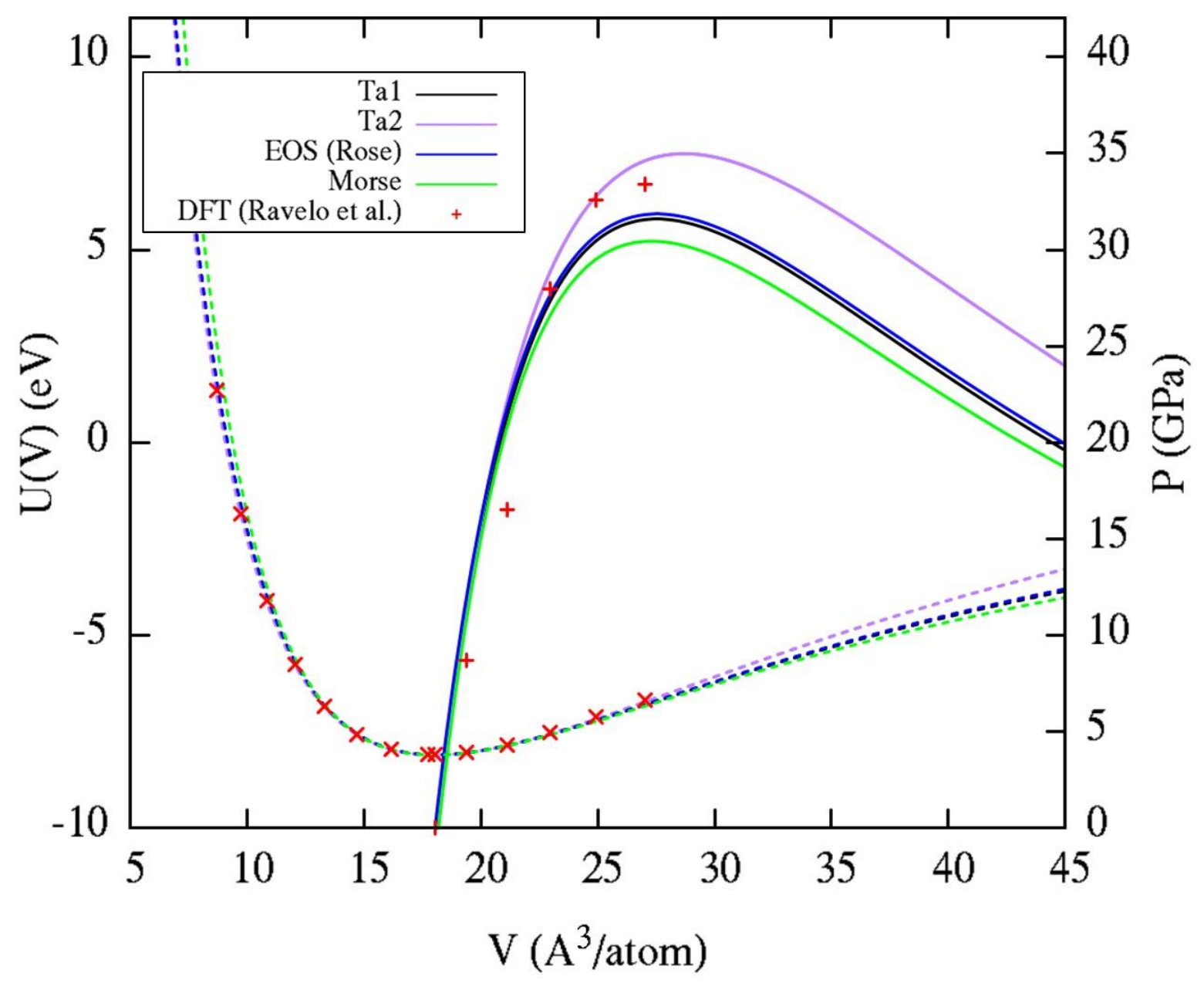

1

2 Figure 14. Simulated cold curves for tantalum. Two tantalum EAM potentials (Ta1 and Ta2 from Ravelo 3 et al.[33]) are compared against an equation of state (EOS) from Rose et al.[81], a Morse potential, and 4 density functional theory (DFT) calculations of Ravelo et al.[33]. Presently derived Morse parameters are: $5 \mathrm{D}_{0}=0.76093, \alpha=1.14113, \mathrm{x}_{0}=3.34342$. This yields $\mathrm{a}_{0}=3.304 \AA, \mathrm{U}_{\text {coh }}=8.1 \mathrm{eV}, \mathrm{B}=194.6 \mathrm{GPa}, \mathrm{C}_{11}=209.9$ $6 \mathrm{GPa}, \mathrm{C}_{12}=\mathrm{C}_{44}=209.9 \mathrm{GPa}$, and $\mathrm{C}_{11} / \mathrm{C}_{12}=1.105$. 
$10^{7} s^{-1}$
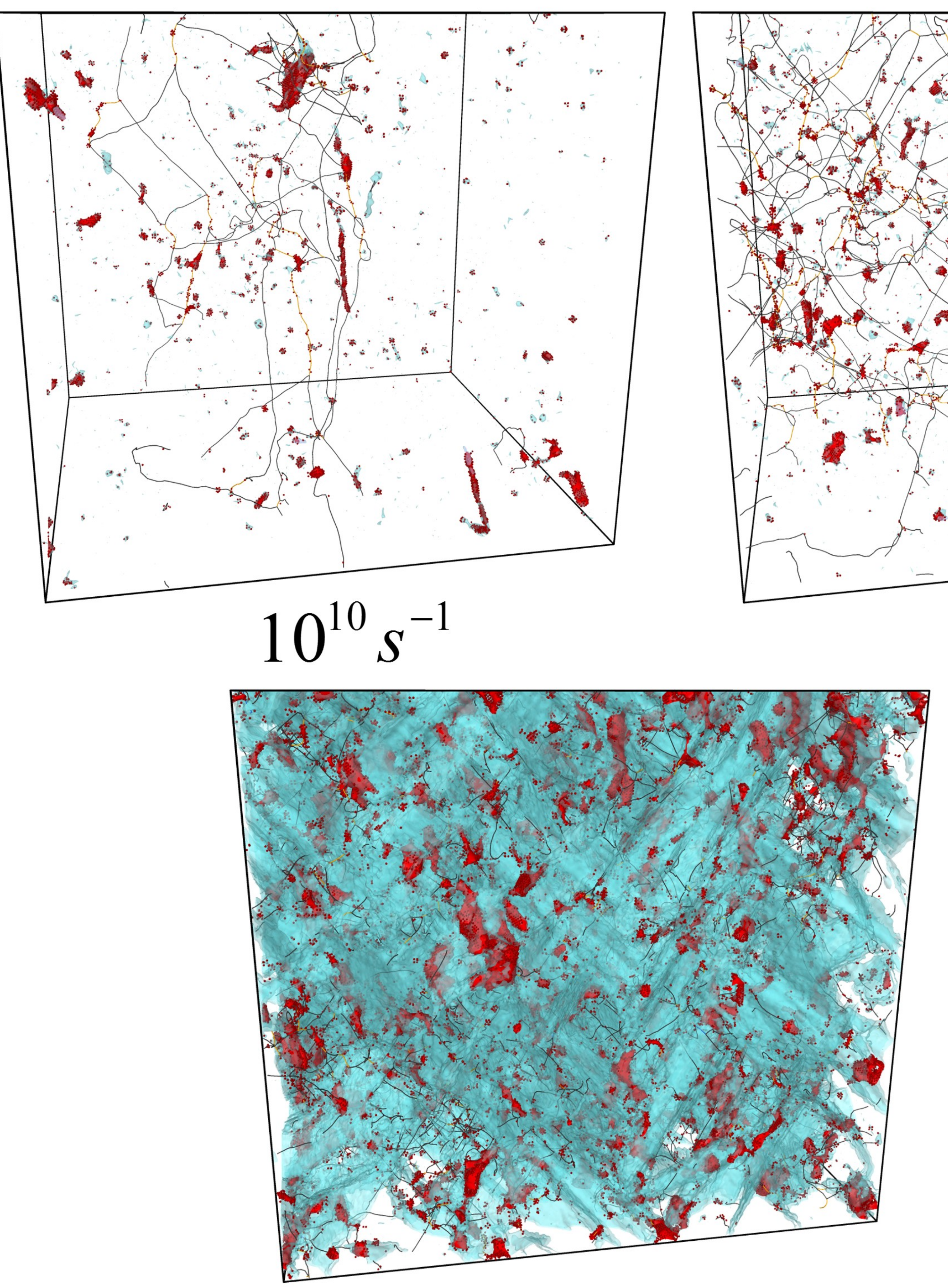
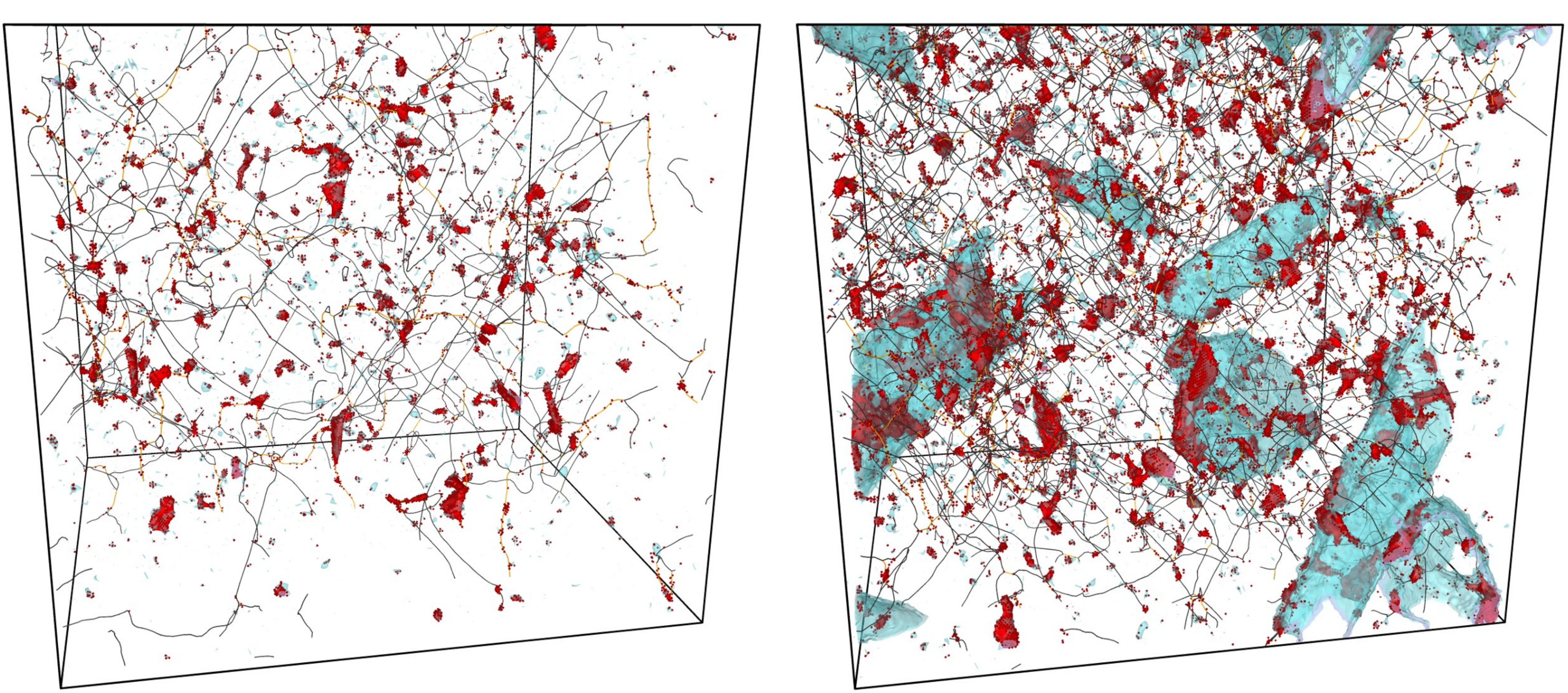

$10^{11} s^{-1}$

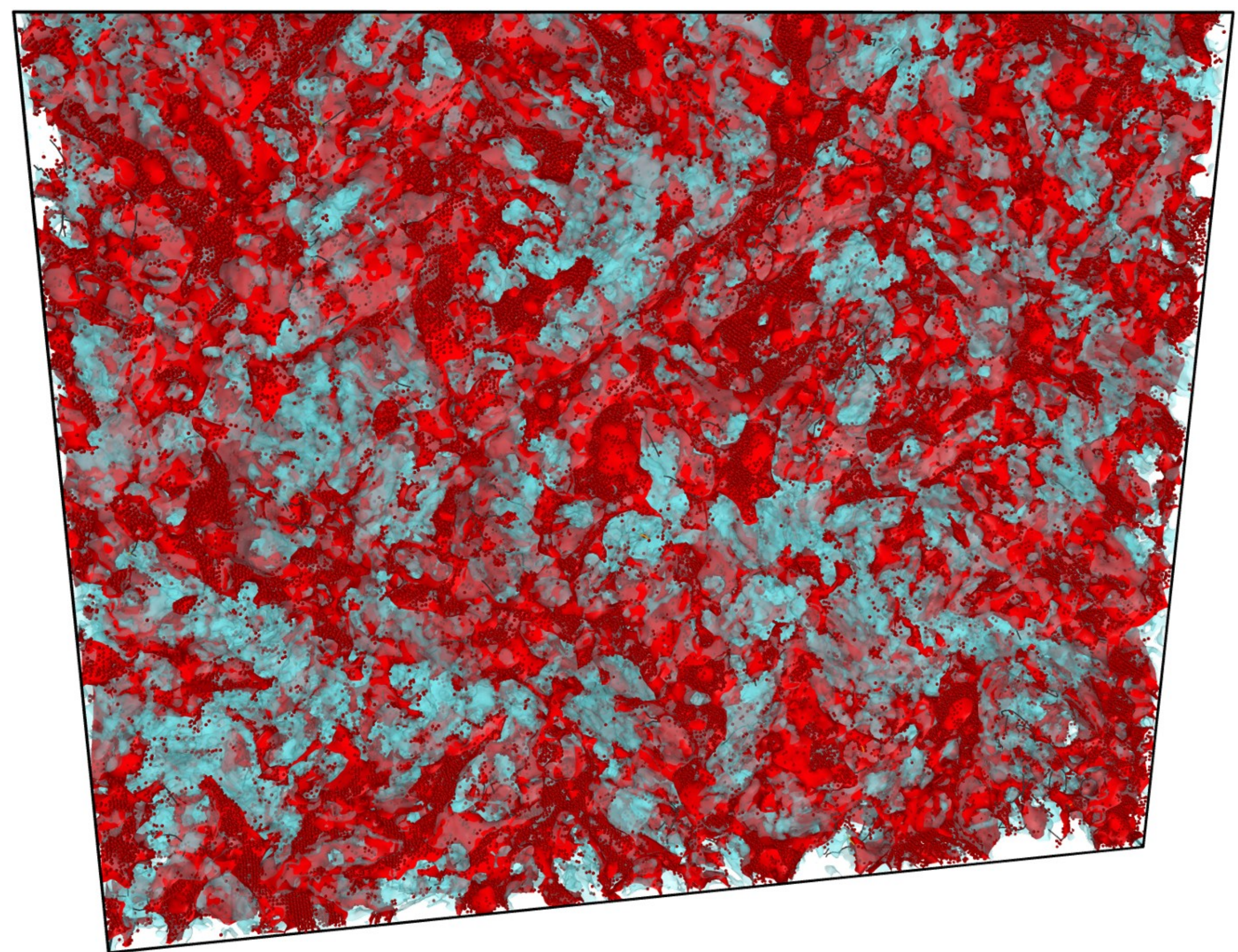

